\title{
PROPAGATION OF LEAKING INTERFACE WAVES
}

\author{
By Robert A. Phinney
}

\begin{abstract}
With simple generalizations of the method due to Rosenbaum (1961) and Phinney (1961), single integral expressions may be written down for the long range pole contributions to the transient signal in a plane seismic waveguide. This method yields expressions for the leaking, or imperfectly trapped waves, and suffers from no restriction on the number of layers or the existence of coupling to one or two half-spaces. When it is applied to the simple interface wave problem of two halfspaces in contact, closed form expressions are obtained describing the propagation of pulses along the interface due to lower sheet poles. The theory is applied to the Lamb problem, the liquid/solid interface, and the solid/solid interface problems. The leaking wave generalizations of the Rayleigh and Stoneley waves are found and a new wave, coupled to the $P$-wave, is demonstrated. The physical importance of leaking interface pulses is shown to be in their coupling to the normal or leaking oscillations of layered structures.
\end{abstract}

\section{TABLE OF SYMBOLS}

\begin{tabular}{|c|c|c|}
\hline Symbol & Definition & EQUATION REFERENCE \\
\hline$a_{i}$ & coefficients in expansion of $f$ & - \\
\hline$A$ & amplitude of source & 1 \\
\hline$b_{i}$ & coefficients in expansion of $g$ & 一 \\
\hline$C, C^{\prime}$ & contour of integration & fig. 2 \\
\hline$c$ & any solution of equation (4) where $c=\omega / \hbar$ & 6 \\
\hline$c_{i}$ & compressional wave velocity in medium $i$ & fig. 1 \\
\hline$E$ & energy in delta-function source & 9 \\
\hline$f$ & period function & 1,4 \\
\hline$g$ & response function & 1 \\
\hline$h$ & source-interface separation & fig. 1 ; eq. 7 \\
\hline$k$ & wave number in horizontal $(r)$ direction & 1 \\
\hline$L_{n}$ & $\begin{array}{l}\text { damping constant of modal oscillations (see } \\
\text { paper I and Rosenbaum, 1960) }\end{array}$ & \\
\hline & $=\operatorname{Im}\left(\omega_{n}^{c}\right)-(r / t) \operatorname{Im}\left(k_{n}^{c}\right)$ & 3 \\
\hline$m$ & $\begin{array}{l}\text { index of halfspace }=\text { number of media in } \\
\text { layered structure }\end{array}$ & 一 \\
\hline$m(c)$ & $\begin{array}{l}\text { excitation function for interface wave prob- } \\
\text { lem }\end{array}$ & 7 \\
\hline$n$ & $\begin{array}{l}\text { subscript indicating evaluation at a root of } \\
\text { the period equation }\end{array}$ & 2 \\
\hline$N$ & $2+$ homogeneous degree of $(g / f)$ & 7,14 \\
\hline$p$ & $\begin{array}{l}\text { order of Bessel function in expression for field } \\
\text { variable; usually zero or one }\end{array}$ & 5 \\
\hline$q_{0}$ & $\begin{array}{l}\text { horizontal displacement on surface of free } \\
\text { halfspace }\end{array}$ & 19 \\
\hline $\begin{array}{l}Q \\
Q_{n}{ }^{c}\end{array}$ & $\begin{array}{l}r-c t+\operatorname{Im} \gamma(c) \\
\text { excitation function of saddle point formula; }\end{array}$ & 10 \\
\hline
\end{tabular}




\begin{tabular}{|c|c|c|}
\hline Symbor & $\begin{array}{l}\text { DEFTNITION } \\
\text { (usage conforms to paper I; appears here } \\
\text { only once) }\end{array}$ & EQUATION REFERENCE \\
\hline$r$ & $\begin{array}{l}\text { horizontal coordinate in circular cylindrical } \\
\text { coordinates }\end{array}$ & 1 \\
\hline$s$ & $=i \omega:$ Laplace transform variable & 1 \\
\hline$t$ & time & 1 \\
\hline$v$ & $\operatorname{Re}(c)$ & 15 \\
\hline$v_{i}$ & $\begin{array}{l}\text { body wave velocity defined by } v_{i}<v_{i+1} \\
\text { equals some } c_{j} \text { or } \beta_{j} \\
\text { vertical displacement on surface of free half- }\end{array}$ & table 2 \\
\hline$\omega_{0}$ & space & 20 \\
\hline$x(t)$ & $\begin{array}{l}\text { field variable, such as pressure, displacement, } \\
\text { stress, etc. }\end{array}$ & 1 \\
\hline$y_{1}$ & $\operatorname{Re} \gamma(c)$ & 15 \\
\hline$y_{2}$ & $\operatorname{Im} \gamma(c)$ & 15 \\
\hline$z$ & $\begin{array}{l}\text { axial coordinate in circular cylindrical co- } \\
\text { ordinates }\end{array}$ & - \\
\hline$\alpha_{i}$ & $\sqrt{k^{2}-\omega^{2} / c_{i}^{2}}=\sqrt{k^{2}+s^{2} / c_{i}{ }^{2}}$ & introduction \\
\hline$\alpha_{i}^{\prime}$ & $\sqrt{k^{2}-\omega^{2} / \beta_{i}^{2}}=\sqrt{k^{2}+s^{2} / \beta_{i}^{2}}$ & introduction \\
\hline$\beta_{i}$ & shear velocity in medium $i$ & - \\
\hline$\gamma(c)$ & $\sqrt{1-c^{2} / c_{i}^{2}}$ & 9 \\
\hline$\delta$ & rotation angle between $k$-plane and $\xi$-plane & 10 \\
\hline$\xi$ & $k e^{-i \delta}$ & 10 \\
\hline$\eta$ & $\operatorname{Im}(c)$ & 9 \\
\hline$\theta$ & time decay constant of source & 1 \\
\hline$\nu_{i}$ & $\sqrt{k^{2}-\omega^{2} / v_{i}^{2}}$ & table 2 \\
\hline$\rho_{i}$ & density of medium $i$ & - \\
\hline$\sigma_{i}$ & Poisson constant of medium $i$ & - \\
\hline$\psi(c)$ & phase of $m(c)$ & 13 \\
\hline$\phi_{0}, \phi$ & 一 & 14 \\
\hline$\omega$ & angular frequency: $=-i s$ & -- \\
\hline
\end{tabular}

\section{INTRODUCTION}

In this paper we make it possible to apply recent developments in leaking mode theory to a fairly broad class of seismic waveguides. What is desired is a simple technique for investigating the complex roots of the period equation and interpreting them in light of an appropriate representation of the solution. We shall first state two generalizations which make it possible to use formulae obtained in an earlier paper for this purpose. In the body of the paper we treat the interface wave problem of two halfspaces in contact on a plane boundary. This is done by reducing the single integral obtained by general considerations to a closed expression representing propagation of a leaking interface pulse. The complex roots of the appropriate period equation may now be numerically evaluated, and the velocity and shape of the pulse then follow.

In a previous paper (Phinney, 1961), which we designate as I, we have deduced 
the leaking, or attenuated, modes of a seismic waveguide consisting of a liquid layer coupled to a solid halfspace. The method (Rosenbaum, 1960) consists of writing the response to a transient point source $\left(x_{0}(t)=e^{-t / 6}\right)$ as a double integral

$$
x(t)=\frac{A}{\pi i} \int_{\lambda i-\infty}^{\lambda+i \infty} \frac{e^{s t}}{s+\theta^{-1}} d s \int_{0}^{\infty} J_{0}(k r) k \frac{g(s, k)}{f(s, k)} d k
$$

where $s$ is the Laplace transform variable, $k$ the wave number in the horizontal coordinate, and $x(t)$ is some field variable such as pressure or displacement. The functions $g(s, k)$ and $f(s, k)$ are determined by applying boundary conditions at the plane interfaces bounding the media.

If we let the index $m$ refer to the halfspace, then $\alpha_{m}=\sqrt{k^{2}+\left(s^{2} / c^{2}\right)}$ and $\alpha_{m}{ }^{\prime}=\sqrt{k^{2}+\left(s^{2} / \beta_{m}{ }^{2}\right)}$, which occur in $g$ and $f$, give rise to branch points in the $s$-plane. Cuts are introduced, joining the branch points to $i \infty$ along the imaginary $s$-axis. The $s$-integration in (1) may be performed by rewriting it as a set of residue contributions from poles lying on the sheet of integration and two branch line integrals arising from integration around the cuts. By deformation of the branch line contours onto lower Riemann surfaces, the branch line integrals are decomposed into (a) residue contributions from complex zeros of $f$ and (b) pulse terms which are interpreted as ray arrivals at the body wave velocities. All the residue contributions from both the top sheet and from the relevant lower sheets have the same form and may be written symbolically as a single integral

$$
x(t)=4 A \sum_{n=0}^{\infty} \int_{S_{\mathrm{I}, \mathrm{II}, \mathrm{III}}} J_{0}(k r) k \frac{e^{s_{n} t}}{s_{n}+\theta^{-1}}\left\{\frac{g}{\partial f}\right\}_{n} d k
$$

where $S_{I, I I, I I I}$ are contours on three Riemann sheets. When this expression is evaluated by a saddle point method in the complex $k$-plane, a representation of the solution at long ranges may be written down. See (3). It expresses the oscillatory contributions at long range in terms of a cosine term and a damped exponential. The subseript $n$ denotes evaluation at a root $s_{n}(k)$ of the period equation $f\left(s_{n}, k\right)=0$. The superscript $c$ denotes evaluation at a saddle point where $r / t=$ $d \omega_{n} / d k .(s=i \omega)$

$$
x(t)=\left|Q_{n}{ }^{c}\right| e^{-L_{n} t} \cos \left[\left(\operatorname{Re} \omega_{n}^{c}\right) t-\left(\operatorname{Re} k_{n}^{c}\right) r+\Omega\right]
$$

\section{Generalizations of the Theory}

We now summarize two results which may be obtained by generalizing the analysis in $I$.

(1) The liquid layer/solid halfspace problem of I differs from the liquid layer/ liquid halfspace problem treated by Rosenbaum (1960) in that the integrand $g / f$ contains two branch cuts instead of one. More general surface waveguides consisting of a stack of solid and liquid layers overlying a solid halfspace still only involve two branch cuts. We may write $f$ and $g$ in the form: $f=a_{0}+a_{1} \alpha_{m}+$ 
$a_{2} \alpha_{m}{ }^{\prime}+a_{3} \alpha_{m} \alpha_{m}{ }^{\prime}: g=b_{0}+b_{1} \alpha_{m}+b_{2} \alpha_{m}{ }^{\prime}+b_{3} \alpha_{m} \alpha_{m}{ }^{\prime}$, where the $a_{i}$ and $b_{i}$ do not involve the branch points. If this form is used in the analysis in $\mathrm{I}$, the result follows that all quantities transform exactly as they did in the particular case in I. The single integral (2) now expresses the pole contributions from all sheets. The saddle point solution (3) now may be applied to this general layered problem, provided only that a saddle point exists and is "accessible" to the initial contour of (2).

(2) If the waveguide under consideration is bounded by halfspaces on both top and bottom, the integrand in (1) will involve up to 4 branch lines. In I we obtained the result shown, which states the values of time for which each Riemann sheet is appropriate:

TABLE 1

\begin{tabular}{r|c|c}
\hline & $\operatorname{Re} \alpha_{2}$ & $\operatorname{Re} \alpha_{2}^{\prime}$ \\
\hline$r / \beta_{2}>t>r / \beta_{2}$ & + & + \\
$0>t>r / c_{2}$ & + & - \\
0
\end{tabular}

To generalize to 3 or 4 branch lines, it is necessary to trace the way in which the period function, $f$, is transformed as the branch line integrals in I are manipulated. The essence of the matter is that each branch line integral is deformed in the s-plane in such a way that the line integral converges most rapidly. When this is done, the resulting line integral and pole contributions are found on some particular "lower" Riemann sheet. Each branch line integral, consisting of two terms, gives rise in this way to pole contributions from two lower sheets; analysis of the conditions which ensure convergence on an arc at $\infty$ gives the range of $t$ which is appropriate to the poles from each sheet, as illustrated in the table above for the 2 branch line problem.

The result for 4 branch lines is stated in table 2 . In connection with the interface wave problem we shall write the restrictions of table 2 in more specific form.

TABLE 2

Two halfspaces in contact, having 4 body wave velocities: $v_{1}<v_{2}<v_{2}<v_{2}$, without specification as to type. Setting $\nu_{i}=\sqrt{k^{2}-\omega^{2} / v_{i}^{2}}$, specification of the sign of the real part of each of the $\nu_{i}$ specifies the Riemann surface appropriate to any given signal velocity according to the table.

\begin{tabular}{c|c|c|c|c}
\hline & $\operatorname{Re} \nu_{1}$ & $\operatorname{Re} \nu_{2}$ & $\mathbf{R} \nu_{3}$ & $\operatorname{Re} \nu_{4}$ \\
\hline $0<r / t<v_{1}$ & + & + & + & + \\
$v_{1}<r / t<v_{2}$ & - & + & + & + \\
$v_{2}<r / t<v_{3}$ & - & - & + & + \\
$v_{3}<r / t<v_{4}$ & - & - & - & + \\
$0<r / t<v_{4}$ & - & - & - & + \\
\hline
\end{tabular}

The first sheet root is real, when it exists. Roots of the period equation lying on lower sheets must be complex with a positive imaginary part if they are to contribute to the signal. (This refers to a complex root $\omega_{n}(k)$, where $k$ is real and positive.) 
In general, we find that when the signal velocity $(r / t)$ lies between two body wave velocities, the relevant Riemann sheet is "upper" for all faster body waves and "lower" for all slower body waves. In all cases, the signal is constrained to vanish prior to the earliest body wave, and the signal after the slowest body wave is de termined by the conventional "top sheet" for all variables.

\section{The General Interface Wave Problem}

We now restrict ourselves to the case of two halfspaces in contact on a plane interface. Three types of problems may be considered (see fig. 1):

(A) A solid halfspace with vanishing stresses on the surface (Lamb's problem).

(B) A solid halfspace in contact with a liquid halfspace.

(C) Two solid halfspaces in welded contact.

Excitation functions and period equations for these problems are well known, since they are derivable from steady-state considerations. A fairly complete collection of results for various types of source is found in Ewing, Jardetzky, and Press (1957).

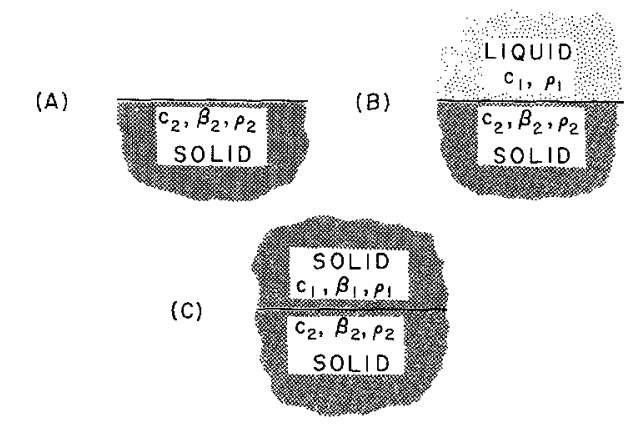

FIG. 1. Three classes of interface wave problem.

In this paper we consider a point source near the interface, and use results given in the reference. Period equations for the three cases are as follows:

$$
\begin{aligned}
\text { (A) } f_{A}= & \left(2 k^{2}-\frac{\omega^{2}}{\beta_{2}{ }^{2}}\right)^{2}-k^{2} \alpha_{2} \alpha_{2}{ }^{\prime} \\
\text { (B) } f_{B}= & \frac{\rho_{1} \omega^{4} \alpha_{2}}{\rho_{2} \beta_{2}{ }^{4}}+\alpha_{1} f_{A} \\
\text { (C) } f_{C}= & k^{2}\left[\frac{\mu_{2}}{\mu_{1}}\left(\frac{\omega^{2}}{\beta_{2}{ }^{2}}-2 k^{2}\right)+\left(2 k^{2}-\frac{\omega^{2}}{\beta_{1}{ }^{2}}\right)\right]^{2} \\
& -\alpha_{1} \alpha_{1}{ }^{\prime}\left[2 k^{2}\left(1-\frac{\mu_{2}}{\mu_{1}}\right)+\frac{\mu_{2}}{\mu_{1}} \frac{\omega^{2}}{\beta_{2}{ }^{2}}\right]^{2}-\alpha_{2} \alpha_{2}{ }^{\prime}\left[2 k^{2}\left(\frac{\mu_{2}}{\mu_{1}}-1\right)+\frac{\omega^{2}}{\beta_{1}{ }^{2}}\right]^{2} \\
& \quad-\left(\alpha_{1} \alpha_{2}{ }^{\prime}+\alpha_{2} \alpha_{1}{ }^{\prime}\right) \frac{\omega^{4} \mu_{2}}{\beta_{1}{ }^{2} \beta_{2}{ }^{2} \mu_{1}}+4 k^{2}\left(\frac{\mu_{2}}{\mu_{1}}-1\right)^{2} \alpha_{1} \alpha_{2} \alpha_{1}{ }^{\prime} \alpha_{2}{ }^{\prime}
\end{aligned}
$$


If a transient point source $x_{0}(t)=A e^{-t / \theta}$ is excited at a distance $h$ from the interface, then the solution is represented as the double integral:

$$
x=\frac{2 A}{\pi i} \int_{\lambda-i \infty}^{\lambda+i \infty} \frac{e^{s t}}{s+\bar{\theta}^{-1}} d s \int_{0}^{\infty} \frac{g(s, k)}{f(s, k)} k J_{p}(k r) d k
$$

where $p$ is zero or one depending on the field variable of interest, $f$ is one of the period functions (4), and $g$ contains the dependence on $h$ and $z$.

By a method due to Cagniard it is possible to obtain an exact closed form solution for all ranges. Cagniard (1939) investigated the solid/solid problem, Garvin (1956) and Gilbert (1956) solved the Lamb problem, and Strick (1959) treated the liquid/solid interface problem. These authors found the various waves long known from asymptotic evaluation of the solution in the steady state. $P$ and $S$ waves arise from integration near a branch point, while the familiar surface waves are due to a pole lying on the sheet of integration. As is customary, this sheet is defined by the condition that $\operatorname{Re} \nu_{i}>0$ for all the radicals $\nu_{i}$; this is brought about by the requirement that the steady state solution die off exponentially away from the interface. Surface waves due to "top sheet" poles may be categorized as follows: (A) the Rayleigh wave, having a velocity between $0.86 \beta_{2}$ and $0.96 \beta_{2}$, (B) the Stoneley wave, having a velocity near $0.99 c_{1}$ for typical models, and (C) the Stoneley wave, with a velocity less than either $\beta_{1}$ or $\beta_{2}$, but greater than the Rayleigh velocity in the faster medium. The Stoneley wave $(C)$ exists only for a very restricted class of models. These remarks are not intended to be complete, but only to characterize. For a complete discussion of the Stoneley wave for (B) and (C), references are given in the bibliography.

Various investigators have suggested the existence of partially trapped interface waves, representing a generalization of the Rayleigh and Stoneley waves cited. Strick (1959) demonstrated a generalized Rayleigh wave for case (B), with a velocity equal to that of the comparable undamped Rayleigh wave (A), but decaying in time by coupling to $P$-waves in the liquid. Gilbert and Laster (1961) have found a partially trapped wave in the Lamb problem, existing in solids with a high Poisson constant. They have also located a leaking Stoneley wave for case $(\mathrm{C})$, when the model parameters do not permit a true Stoneley wave to exist. All of these pseudo-waves are caused by poles of the response which lie on Riemann sheets other than the principal sheet of integration. By their proximity to the branch cuts (in the Cagniard formulation) they affect the form of the solution obtained by integration around the cuts, thus generating a contribution to the signal, despite their location on a lower sheet. Investigations of the sort cited are equipped to delineate exact seismogram shapes, but prove tedious in many cases without shedding proportionate light on the physical nature of the signals. In this paper we treat the interface wave problem in a more traditional way, obtaining asymptotic formulae for the pulse shape by direct attack on the double integral solution (5). The results cited in the introduction make it possible to do so with a precision which brings the lower sheet contributions into sharp focus, instead of leaving them implicit in the branch line integrals as has been done up to now.

Our starting point is the single integral formula (2), a rather general tool which 
is easily applied to the cases we are considering. An asymptotic representation of the pulse due to each lower sheet pole arises directly from (2) by exact evaluation of the integral resulting from the long distance approximation to the Bessel function. In this approximation each wave has a constant velocity, given by the real part of the root $c$ of the period equation. We may now view the leaking interface waves as free vibrations of the interface in a more general sense than that which describes ordinary undamped surface waves. Arising from so-called extraneous roots of the period equation, the pseudo-waves make sense physically in terms of plane wave reflection and transmission coefficients. The attractiveness of this method lies in its simplicity; for a given set of layer parameters the complex roots of the period equation may be easily obtained, enabling one to predict the velocity, pulse width, and attenuation (as well as existence) of all the trapped or partially trapped free waves.

For each pole of the integrand which lies on a permissible sheet (tables $1-3$ ) we may write down the single integral obtained from (2):

$$
x=4 A R e \int_{0}^{\infty} J_{p}(k r) \frac{e^{i k c t}}{i k c+\theta^{-1}}\left(\frac{g i}{\partial f}\right)_{\omega=k c} k d k
$$

Since the period equation (4) is homogeneous in $\omega$ and $k$, it is possible to write $f=f(\omega, k)=f(k c, k)=k^{i} f(c, 1)=0$. Thus roots of the period equation may be regarded as single numbers $c$, rather than functions $\omega_{n}(k)$. Henceforth, appearance of the letter $c$ implies that it is a root of the period equation. The integrand in (6) is the result of the substitution $\omega=k c$. Since $g$ is also homogeneous, the expression in parentheses reduces to one of the form $k^{N-1} m(c)$, where $m(c)$ is a complex function of a complex $c$. If the source or receiver are not on the interface, exponential factors of the form

$$
\exp \left(\sqrt{k^{2}-\frac{\omega^{2}}{c_{i}^{2}}}|z|\right) \text { or } \exp \left(\sqrt{k^{2}-\frac{\omega^{2}}{c_{i}^{2}}} h\right)
$$

will also result (from the function $g$ ). It is sufficiently general for our purposes to assume $z$ is zero and $h$ positive, with the source compressional in nature and lying in medium $i$. Now (6) may be written:

$$
x=4 A \operatorname{Re}\left\{m(c) \int_{0}^{\infty} k^{N} J_{p}(k r) \frac{e^{i k c t}}{i k c+\theta^{-1}} e^{-k h \sqrt{1-\left(c^{2} / c_{i}^{2}\right)}} d k\right\}
$$

We now make the only approximation in the analysis. It is the conventional long distance representation of the Bessel function, under the assumption of no sources at infinity:

$J_{p}(k r)=H_{p}{ }^{(1)}(k r)+H_{p}{ }^{(2)}(k r) \rightarrow H_{p}{ }^{(2)}(k r)$

$$
\sim \sqrt{\frac{2}{\pi k r}} \exp \left[-i\left(k r-\frac{\pi}{4}-\frac{p \pi}{2}\right)\right]
$$


Then:

$$
x=4 A \operatorname{Re}\left\{\sqrt{\frac{2}{\pi r}} m(c) e^{i(\pi / 2)[p+(1 / 2)]} \int_{0}^{\infty} k^{N-(1 / 2)} \frac{e^{-i k\left(r-c t-i h \sqrt{1-\left(c^{2}-c_{i}^{2}\right)}\right.}}{i k c+\theta^{-1}} d k\right\}
$$

If we assume the source function to be a delta-function in time, then $\theta^{-1} \rightarrow \infty$. We let the source amplitude $A \rightarrow \infty$ as well, so that the source pulse still has finite energy. Setting $E=A \theta$ and $\gamma(c)=\sqrt{1-c^{2} / c_{i}^{2}}$ :

$$
x=4 E \sqrt{\frac{2}{\pi r}} \operatorname{Re}\left\{m(c) e^{i(\pi / 2)[p+(1 / 2)]} \int_{0}^{\infty} k^{N-(1 / 2)} e^{-i k(r-c t-i h \gamma(c))} d k\right\}
$$
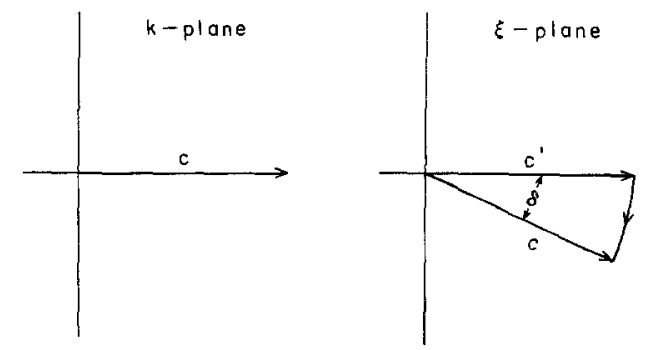

Fig. 2. $k$-plane and $\xi$-plane for integration of (10).

Consider the integral alone: write:

$$
Y=\int_{0}^{\infty} k^{N-(1 / 2)} e^{-i k Q} d k \quad \text { where } \quad Q=r-c t-i h \gamma(c)
$$

This is easily evaluated if the variable $k$ is replaced by $\xi=k e^{-i \delta}$. The effect is a rotation of the $k$-plane. (Fig. 2)

The original contour $C$ in the $k$-plane maps into $C$ in the $\xi$-plane. We now distort $C$ into $C^{\prime}$ along the real $\xi$-axis and along an arc at infinity. $\delta$ is chosen so that $C^{\prime}$ is a steepest descent path, as follows:

$$
\begin{aligned}
& Q=Q_{1}+i Q_{2} \\
& \exp (-i k Q)=\exp \left(-i k Q_{1}\right) \cdot \exp \left(k Q_{2}\right) \\
& \exp \left[-i \xi Q_{1}(\cos \delta+i \sin \delta)\right] \cdot \exp \left[\xi Q_{2}(\cos \delta+i \sin \delta)\right] \\
& \exp \left[-i \xi\left(Q_{1} \cos \delta-Q_{2} \sin \delta\right)\right] \cdot \exp \left[-\xi\left(-Q_{1} \sin \delta-Q_{2} \cos \delta\right)\right]
\end{aligned}
$$

For a steepest descent path $\delta$ is chosen so that $Q_{1} \cos \delta=Q_{2} \sin \delta$. In other words:

$$
\begin{aligned}
& \sin \delta=-Q_{1} /|Q| \\
& \cos \delta=-Q_{2} /|Q|
\end{aligned}
$$

where the - sign assures convergence of the integral. 
Thus:

$$
\exp (-i k Q)=\exp \left[-\xi \frac{Q_{1}^{2}+Q_{2}^{2}}{|Q|}\right]=\exp [-\xi|Q|]
$$

Therefore:

$$
\begin{aligned}
Y & =\int_{0}^{\infty}\left(\xi e^{i \delta}\right)^{N-(1 / 2)} e^{-\xi|Q|} d\left(\xi e^{i \delta}\right) \\
& =e^{i \delta[N+(1 / 2)]} \int_{0}^{\infty} \xi^{N-(1 / 2)} e^{-\xi|Q|} d \xi
\end{aligned}
$$

This integral may be evaluated in terms of the gamma function:

Thus:

$$
\begin{aligned}
& Y=\frac{e^{i \delta[N+(1 / 2)]} \Gamma\left(N+\frac{1}{2}\right)}{|Q|^{N+1 / 2}} \\
& Y=\frac{e^{i \delta[N+11 / 2)} \lambda(N) \sqrt{\pi}}{|Q|^{N+(1 / 2)}}
\end{aligned}
$$

Substituting this in (9), we get:

$$
x=4 E \sqrt{\frac{2}{\pi r}} \operatorname{Re}\left\{m \left(c\left(e^{i \delta[N+(1 / 2)]} e^{i \pi / 2[p+(1 / 2)]} \lambda(N) \sqrt{\pi}|Q|^{-[N+(1 / 2)]}\right\}\right.\right.
$$

If $m(c)$ is cast in polar form, $|m(c)| e^{i \psi(c)}$, then

$$
x=\frac{4 E \sqrt{2}}{r^{1 / 2}\left|Q_{r, \ell}\right|^{N+(1 / 2)}}|m(c)| \lambda(N) \cos \left[\psi(c)+\frac{\pi}{2}\left(p+\frac{1}{2}\right)+\left(N+\frac{1}{2}\right) \delta_{r, t}\right]
$$

where:

$$
\begin{array}{ll}
\delta & =\frac{3}{2} \pi-\text { phase }(Q) \\
Q & =(r-c t)-i h \gamma(c) \text { where } c \text { is in general complex } \\
\lambda(N) & =\frac{\Gamma\left(N+\frac{1}{2}\right)}{\sqrt{\pi}} \\
N & =2+\text { homogeneous degree of } g / f \\
p & =\text { order of Bessel function } \\
m(c) & =\text { excitation function depending on model and source. }
\end{array}
$$

By subscripts $r, t$ it is suggested that $Q$ and $\delta$ are functions of the time and horizontal range. It is this dependence, in fact, which shapes the pulse. Equation (13) may be cast in a more convenient form depending explicitly on the phase of $Q$ (which we denote by $\phi$ ).

$$
x=\frac{4 E \sqrt{2}}{r^{1 / 2}\left|Q_{r, t}\right|^{N+(1 / 2)}}|m(c)| \lambda(N) \cos \left[\phi_{0}-\left(N+\frac{1}{2}\right) \phi_{r, t}\right]
$$


where

$$
\phi_{0}=\psi(c)+\pi+(\mathrm{p}-N) \frac{\pi}{2}
$$

We shall use (14) to describe the shape of the pulse propagated along an interface due to complex poles, $c$, of the period equation. It is, therefore, necessary at this point to write down in tabular form the restrictions on the Riemann sheets for various values of $t$. These are taken from Table 2 , or a similar table for two or three branch lines.

(A) Free solid halfspace: $\beta_{2}<c_{2}$

$$
\begin{aligned}
0<r / t & <\beta_{2} \\
\beta_{2}<r / t & <c_{2}
\end{aligned}
$$

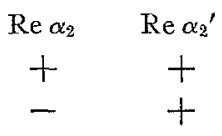

For all three problems the "lower" sheet for all variables (last line in Table 2) is identical to the "top" sheet. This is seen by inspection of the period equations (4). It is therefore not relevant to the description of the pole contributions.

(B) Liquid halfspace/solid halfspace:

$$
c_{1}<\beta_{2}<c_{2}
$$

$0<r / t<c_{1}$ (Stoneley wave)

$c_{1}<r / t<\beta_{2}$ (Pseudo-Rayleigh)

$\beta_{2}<r / t<c_{2}$ (Pseudo- $P$ wave)

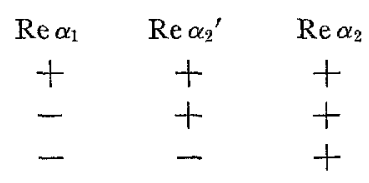

$$
\beta_{2}<c_{1}<c_{2}
$$

$0<r / t<\beta_{2}$

$\beta_{2}<r / t<c_{1}$

$c_{1}<r / t<c_{2}$

$$
\beta_{2}<c_{2}<c_{1}
$$

$0<r / t<\beta_{2}$

$\beta_{2}<r / t<c_{2}$

$c_{2}<r / t<c_{1}$

(C) Solid halfspace/solid halfspace:

$\begin{array}{lll}+ & + & + \\ + & - & + \\ - & - & +\end{array}$

$0<r / t<\beta_{1}$

$\beta_{1}<r / t<c_{1}$

$c_{1}<r / t<\beta_{2}$

$\beta_{2}<r / t<c_{2}$

$$
\beta_{1}<c_{1}<\beta_{2}<c_{2}
$$

$\begin{array}{cccc}\operatorname{Re} \alpha_{1}{ }^{\prime} & \operatorname{Re} \alpha_{1} & \operatorname{Re} \alpha_{2} & \operatorname{Re} \alpha_{2} \\ + & + & + & + \\ - & + & + & + \\ - & - & + & + \\ - & - & - & +\end{array}$




$$
\beta_{1}<\beta_{2}<c_{1}<c_{2}
$$

$0<r / t<\beta_{1}$

$\beta_{1}<r / t<\beta_{2}$

$\beta_{2}<r / t<c_{1}$

$c_{1}<r / t<c_{2}$

$\begin{array}{llll}+ & + & + & + \\ - & + & + & + \\ - & + & - & + \\ - & - & - & +\end{array}$

$\beta_{1}<\beta_{2}<c_{2}<c_{1}$ $0<r / t<\beta_{1}$

$\beta_{1}<r / t<\beta_{2}$

$\beta_{2}<r / t<c_{2}$

$c_{2}<r / t<c_{1}$

$\begin{array}{llll}+ & + & + & + \\ - & + & + & + \\ - & + & - & + \\ - & + & - & -\end{array}$

All other combinations of body velocities are either trivial or physically inadmissible. Henceforth, we refer to a Riemann sheet by a sequence of +'s and -'s: e.g. $(-+),(-++)$, or $(-+-+)$.

\section{Typical Pulse Shapes Predicted by (14)}

We now inspect the pulse representation (14) in enough detail to show its relation to the physies of the problem and to demonstrate its agreement with known results for undamped surface waves. Written in terms of real quantities, $Q$ has the form:

$$
Q=\left(r-v t+h y_{2}\right)-i\left(\eta t+h y_{1}\right)
$$

where:

$$
\begin{array}{ll}
v=\operatorname{Re} c & y_{1}=\operatorname{Re} \sqrt{1-c^{2} / c_{i}^{2}} \\
\eta=\operatorname{Im} \mathrm{c} & y_{2}=\operatorname{Im} \sqrt{1-c^{2} / c_{i}^{2}}
\end{array}
$$

Case (B) is taken as representative. We take a model typical of water in contact with a solid, with the source located in the water layer: $c_{1}=1.0, c_{2}=3.0, \beta_{2}=$ 1.732 , and $\rho_{2}=2 \rho_{1}$ (a Poisson solid):

\begin{tabular}{cccl} 
sheet & $\mathrm{v}$ & $\eta$ & \multicolumn{1}{c}{ type of wave } \\
$(+++)$ & 09860 & - & Stoneley \\
$(-++)$ & 1.6151 & 0.0980 & Pseudo-Rayleigh \\
$(--+)$ & 3.036 & - & Trivial pseudo-P \\
$(--+)$ & 3.917 & - &
\end{tabular}

When Poisson's constant is taken to be 0.355 , the situation is slightly different: $c_{1}=1.0, c_{2}=3.0, \beta_{2}=1.41$, and $\rho_{2}=2 \rho_{1}$ :
$(+++)$
0.966
0.0917
Stoneley
$(-++)$
1.348
0.492
Pseudo-Rayleigh
2.768
Pseudo- $P$

These results are taken from a later section, where the complex phase velocities 
for a representative class of models have been computed and presented in the form of arrival-time diagrams.

The pulse is shaped by (14) as follows. A cylindrical spreading term, $r^{-(1 / 2)}$, which multiplies the pulse amplitude, is identical with that obtained for point source excitation of true surface waves. If we were considering a line source problem, this factor would be missing. The factor $|Q|^{-[N+(1 / 2)]}$ determines the pulse envelope, and $\left(n+\frac{1}{2}\right) \phi$, as an argument of a cosine function, determines oscillation of the signal within the pulse envelope. For all the cases considered in this paper, $N=2$. Higher $N$ corresponds to a field variable obtained by one or more
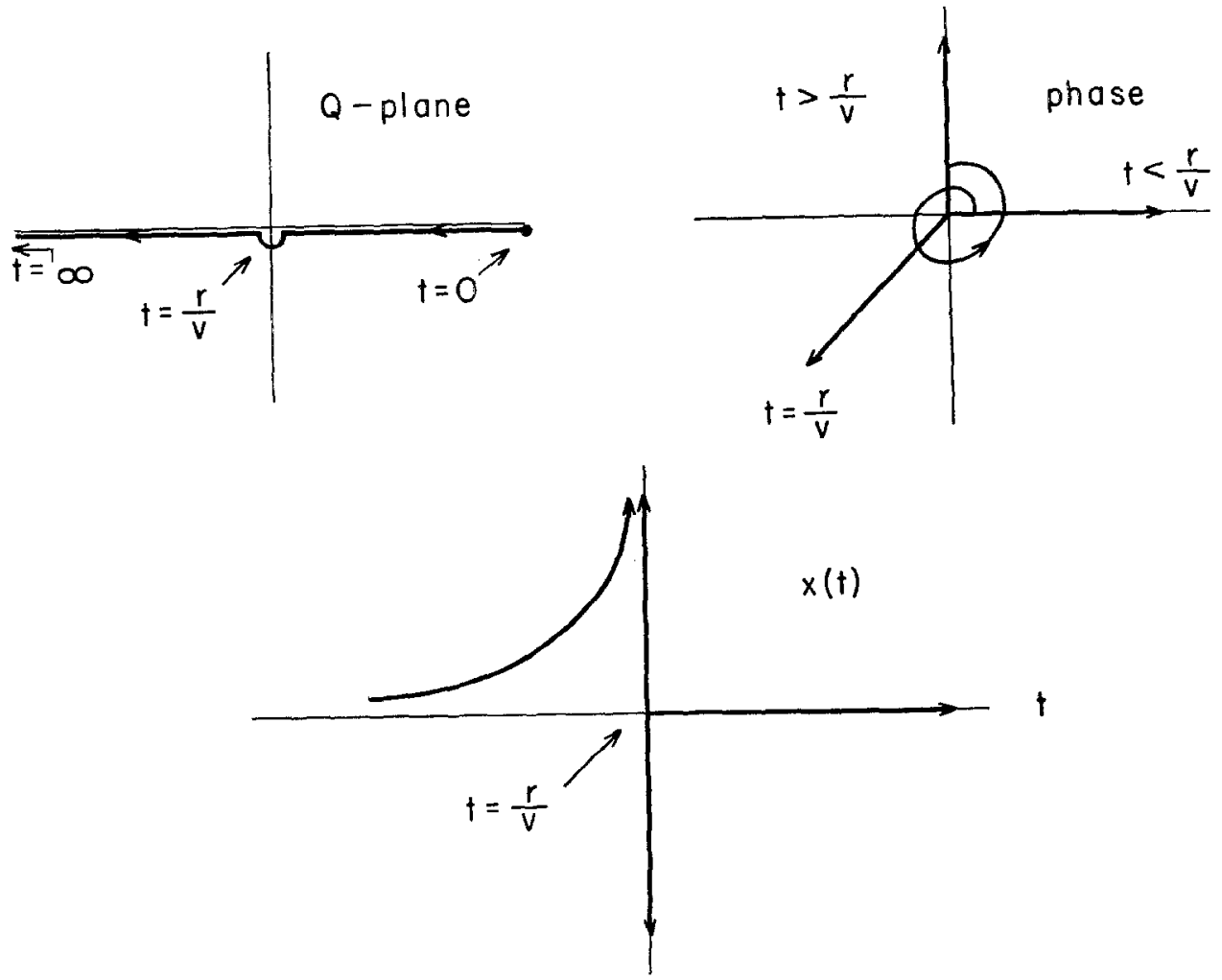

FIG. 3. Q-plane, $\left.\left[\phi_{0}\right]-\left(\frac{5}{2}\right) \phi\right]$, and $x$ as functions of $t$. Stoneley wave: $h=0, \eta=0$.

spatial or time derivatives; its appearance in the phase factor makes sense from this point of view.

Stoneley wave: $h=0, \eta=0 . Q$ vanishes when $t=r / v$, namely at the arrival time of the wave whose velocity $=$ Re $c$. There is, therefore, a singularity at the arrival time of the Stoneley wave. Also, $m(c)$ is real, therefore $\psi(c)=0$; for the pressure signal in the water, $p=0$. Thus $\phi_{0}=\pi+0+0+3 \pi=4 \pi \rightarrow 0$. When $t<r / v, \phi=0$; when $t=r / v, \phi=-\frac{1}{2} \pi$ (assuming a small + imaginary $\gamma_{t}$ ); when $t>r / v, \phi=-\pi$. Figure 3 shows: (1) $Q$ as a function of $t$ (in the complex plane), (2) a polar diagram of phase, (3) and the consequent pulse shape, obtained by applying the envelope function and the cosine operation. 
The singularity is, of course, a result of taking a delta function source in the first place, and is suggestive of the fact that the Stoneley wave is perfectly trapped and that the high frequencies are preserved in transmission. (The pulse shape shown is just that due to the Stoneley pole; when considered as part of an actual seismogram, we must "gate" the pulse on at $t=r / c_{1}$, and superimpose the contribution from the direct body wave. A similar qualification holds for all the pulse shapes we shall derive.)
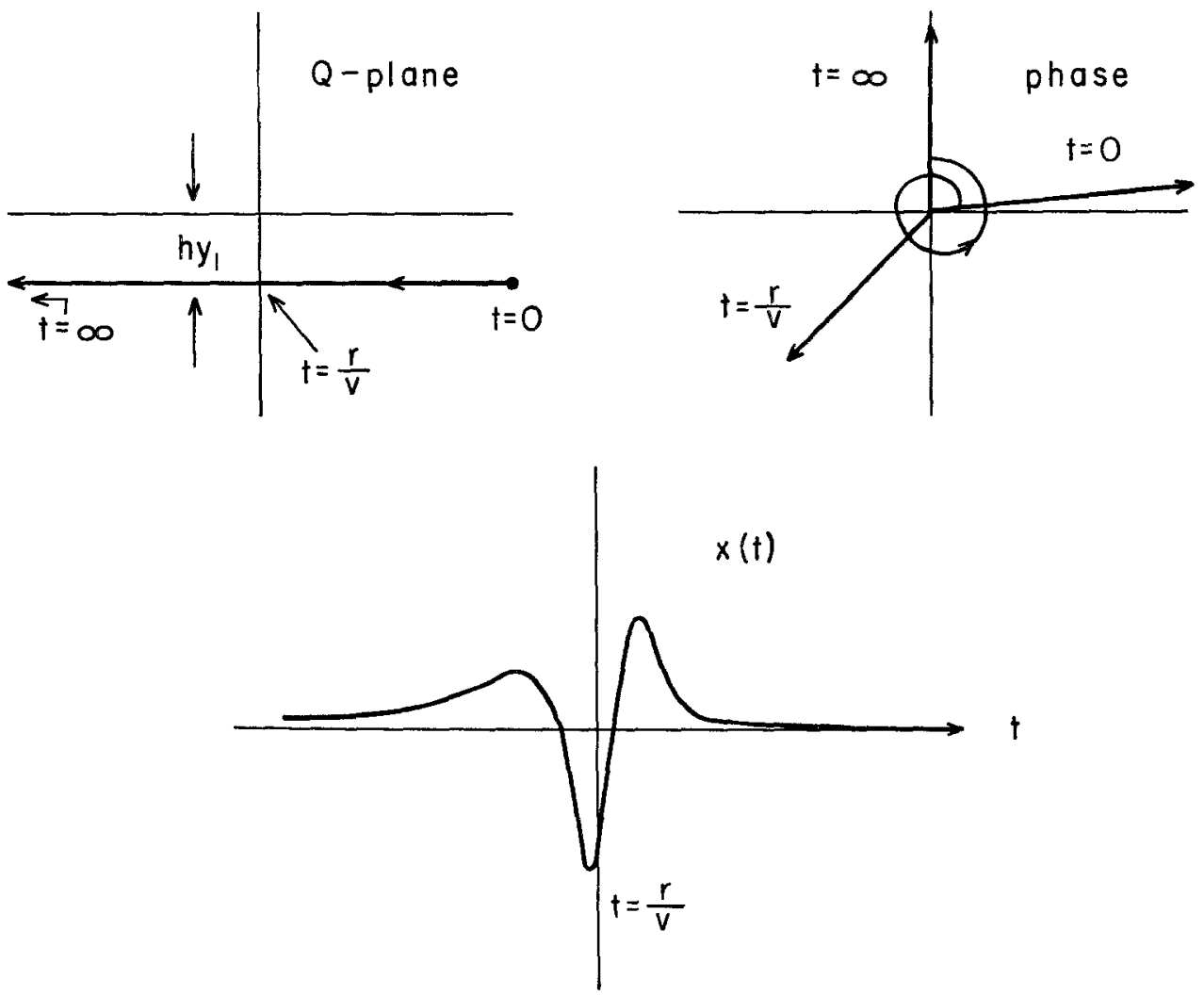

FIG. 4. $Q$-plane, $\left[\phi_{0}\right]$-(这) $\left.\phi\right]$, and $x$ as functions of $t$. Stoneley wave: $h \neq 0, \eta=0$.

When we take into account the separation of the source and interface, $h \neq 0$, $\eta=0$, and $y_{2}=0$ (since $c=v<c_{1}$ ). Figure 4 shows the variation (with $t$ ) of $Q$, $\phi_{0}-\left(\frac{5}{2}\right) \phi$, and $x(t)$. The effect of removing the source from the interface is to remove the high frequencies from the interface wave. The source preferentially excites longer wavelengths; in the same way, a receiver not on the interface does not "see" shorter waves due to their greater localization near the interface.

The preceding has been to demonstrate the use of the representation (14), since the Stoneley wave is no newcomer, and has been thoroughly described in the literature. We now demonstrate its applicability to leaking interface waves. The results to be derived for the pseudo-Rayleigh wave will bear out some of the properties discussed by Strick (1959) in his exact treatment. This is just a Rayleigh wave on 
the solid which loses energy by conversion into compressional waves radiating into the liquid. It therefore looks like an interface wave with respect to the solid, but has the properties of a critically refracted ray in the liquid. Its velocity is essentially that of a Rayleigh wave on a free halfspace. We set $h=0$ in order to isolate the effect of $\eta$ on the pulse shape. Then:

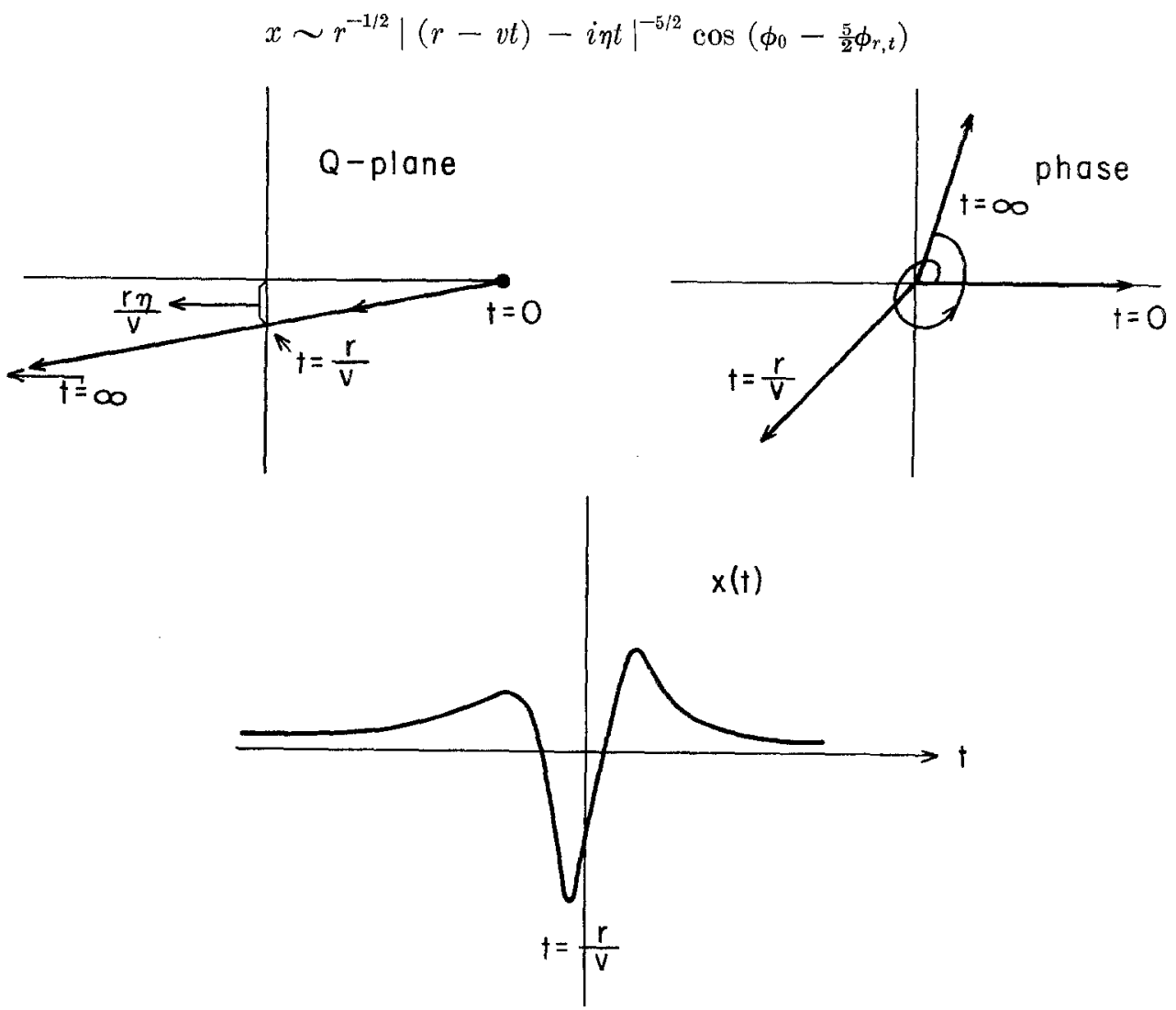

Frg. 5. Q-plane, $\left[\phi_{0}-\left(\frac{5}{2}\right) \phi\right]$, and $x$ as functions of $t$. Pseudo-Rayleigh wave: $h=0, \eta \neq 0$.

To ease the computation slightly, we set $\psi(c)=0$, an assumption which affects the phase of the wavelet, but not the point we wish to make. Fig. 5 shows the construction of the waveform as before. The existence of $\operatorname{Im} c=\eta$ manifests itself in a smoothing of the waveform and a reduction of the maximum amplitude. In other words, the higher frequencies attenuate more rapidly than the lower frequencies. This is as it must be: The absence of a characteristic dimension in the geometry implies, by a similarity argument, that the decay range of a given frequency must be proportional to its wavelength.

For $h \neq 0$, we must take into account $y_{1}+i y_{2}=\sqrt{1-c^{2} / c_{1}^{2}}$. Then $c$ is nearly real, and $\operatorname{Re} c>c_{1}$. Therefore $y_{1}$ is small and negative, while $y_{2}$ is large and positive. The sign choice is due to the fact that we are on the sheet where $\operatorname{Re} \alpha_{1}<0$. Then:

$$
x \sim r^{-1 / 2}\left|\left(r-v t+h y_{2}\right)-i\left(\eta t+h y_{1}\right)\right|^{-5 / 2} \cos \left(\phi_{0}-\frac{5}{2} \phi\right)
$$


In harmonic wave theory, $y_{1}$ describes a wave increasing exponentially away from the interface. Here, its only effect is to decrease the bluntness of the pulse by counteracting the $\eta t$ term. Alternatively, we may say that the existence of a ray path into the liquid permits the source to communicate the high frequencies to the interface more efficiently than was possible for the wholly trapped Stoneley wave (and reciprocally). The factor $y_{2}$ is responsible for a term. which delays the peak amplitude of the pulse a time equal to the time required for the signal to reach the interface along the critical ray feeding the Rayleigh wave in the solid. Thus, while roots of the characteristic equation lying on lower Riemann sheets yield physically impermissible steady-state waves, the result of superposition in the time domain is physically quite reasonable.

The pseudo- $P$ wave, which we shall discuss later in detail, is an intrinsic leaky vibration of a solid halfspace which does not "see" the liquid halfspace in a significant way. Energy is radiated away from the interface as shear energy into the solid and as $P$ waves into the liquid. When $\sigma_{2}=0.355$, for example, the maximum pulse amplitude propagates at a velocity slower than $c_{2}$. The high imaginary part of $c$ suggests that this pulse is very much blunted in comparison with the PseudoRayleigh wave. We recall that this pulse is identically zero prior to $t=r / c_{2}$, and is "gated" on only with the arrival of the initial $P$ phase. When $\sigma_{2}=0.25$, the pair of roots which were complex conjugate for $\sigma_{2}=0.355$ have degenerated to a pair of non-equal real roots whose real parts exceed $c_{2}$. The main body of the corresponding pulse never actually exists, because the signal is constrained to vanish prior to $t=r / c_{2}$; at times shortly after the $P$-wave, however, these poles (particularly the slower one) distort the signal slightly, giving the $P$-wave a slight tail.

In summary: The solution (14) is an asymptotic representation of the pulse propagated along a plane interface due to complex roots of the characteristic equation (4). The results are subject to the constraints of Tables 1 and 2, which denote the permitted Riemann sheets for any $r, t$. As pointed out by Strick (1959), these quasi-surface waves are not always physically separable on experimental or exact theoretical records due to their close association with body phases. The purpose of our demonstration has been to generalize the familiar long range expression of free surface waves to describe partially trapped waves in the same framework. A harmonic theory is, of course, not possible; we may, however, speak of the complex roots of the characteristic equation in the same breath as the real roots. They are interpreted as modes of motion which involve less coupling to body waves (radiation away from the interface) than neighboring motions (in a variational sense).

Having a single framework for both true surface waves and complex surface waves, we hope that the nomenclature may become standardized. The resemblance between the true Rayleigh wave, the Stoneley wave for a liquid/solid interface, and the Stoneley wave for a solid/solid interface is superficial, since they arise by coupling to the lowest body wave velocity in the system, which is different in each case. Now it will be possible to identify corresponding wave types for the 3 types of system; Strick (1959) has already established, for example, the correspondence of the true Rayleigh wave (A) with the pseudo-Rayleigh wave (B).

Mathematically our solution has the following properties:

1. It was not necessary to approximate the second integral beyond using the long 
range representation of the Bessel function. If desired, we could write out further terms in the asymptotic series for the Bessel function and obtain a more precise estimate of the pulse shape. As this is not our object, we defer to the exact solution by the method of Cagniard, should theoretical seismograms be desired.

2. The existence of characteristic roots on lower Riemann sheets is physically permissible in the time domain. The only effect of $\operatorname{Re} \nu_{i}$ (be it + or - ) is to modify the pulse shape slightly. Im $\nu_{i}$, which is connected with radiating body waves, appears in a time delay term which accounts for the separation of the source and the interface.

3. Reciprocity enables us to make the same remarks about a receiver at distance $z$ from the interface as we have made concerning a source at $h$. By superposition we may consider both simultaneously.

4. A pseudo-surface pulse will travel faster than certain of the body velocities and slower than the remainder. The former group will couple to the pseudo-wave as body waves in the appropriate medium. The latter velocities cause the trapped component of the energy. Thus an instrument sensitive to the body potentials with velocities $v_{i}>\operatorname{Re} c$ will see an exponential decrease away from the interface. An instrument sensitive to wave potentials whose velocities $v_{i}<\operatorname{Re} c$ will see body waves feeding (or leaving) the interface at a critical angle $\theta_{i}: \sin \theta_{i}=v_{i} / v$.

In what follows we shall display the complex roots of the period equation in a manner which will be suggestive of the form of the pulse expression (14). The factor $h$ has the effect of modifying and delaying the pulse arrival, but since propagation along the interface is of interest, we set $h=0$. The factor $v$ is the velocity of the maximum pulse amplitude, and $\eta$ causes a broadening and weakening of the pulse. A semi-quantitative measure of the pulse broadening may be obtained: We express the envelope function $|Q|^{-(N+(1 / 2))}$ in terms of the arrival time of the pulse, and solve for the time where the envelope is down by some factor. It is assumed that $(\eta / v)<0.3$, so that $(\eta / v)^{2}$ may be neglected in the result.

$$
\begin{aligned}
x \sim r^{1 / 2} \mid(r-v t)- & \left.i \eta t\right|^{-[N+(1 / 2)]}=r^{-1 / 2} r^{-[N+(1 / 2)]} \cdot\left|\left(1-\frac{v t}{r}\right)-i \frac{\eta t^{-[N+(1 / 2)]}}{r}\right|^{-[N+1)}\left|1-t^{\prime}-i \frac{\eta}{v} t^{\prime}\right|^{-[N+(1 / 2])}=r^{-(N+1)}|P|^{-[N+(1 / 2)]}
\end{aligned}
$$

$t^{\prime}=1$ corresponds to the pulse maximum.

When $t^{\prime}=1,|P|=\eta / v$

When $t^{\prime}=1+\tau,|P|=\sqrt{\tau^{2}+\left(\frac{\eta}{v}\right)^{2}(1+\tau)^{2}}$

This we set equal to $m|P(1)|=m \frac{\eta}{v}$

Solving for $\tau$ and dropping $(\eta / v)^{2}$ in the result, we have an estimate of the halfwidth of the pulse in terms of its arrival time:

$$
\tau \sim\left(\frac{\eta}{v}\right) \sqrt{m^{2}-1}
$$


For $n=2, m=2$ suffices to describe the point at which the pulse has about $\frac{1}{6}$ peak amplitude. We shall assume the formula $\tau=1.7(\eta / v)$ in plotting the halfwidth in figures $6-13$.

Another measure of pulse width in this case is the peak to trough time for the oscillatory center of the pulse Under assumptions like those given above, $0.7(\eta / v)$ is an equivalent estimate, which we shall not plot.

Setting $t^{\prime}=1$ in (16), we get an expression for the decay of the peak amplitude with distance.

$$
x \sim r^{-(N+1)}\left(\frac{\eta}{v}\right)^{-[N+(1 / 2)]}=r^{-1 / 2}\left(\frac{r \eta}{v}\right)^{-[N+(1 / 2)]}
$$

Numerical results: These are presented in the form of an arrival-time diagram. The horizontal axis is the time normalized to the arrival of one of the body waves in the system. The vertical coordinate represents the variation of some function of the densities and body velocities; in this way we are able to show the behavior of the interface waves for a representative class of models.

The arrival of the various body waves and true interface waves is represented by solid lines, and the pseudo-waves are indicated by the arrival of the pulse maximum. Shading indicates the pulse width of the pseudo-waves according to (17). The areas between the body waves are labeled with the $(-+++)$ notation to denote the relevant Riemann sheet for interface wave propagation.

\section{The Lamb Problem}

From equations (2-86) in Ewing, Jardetzky, and Press (1957), we may write the surface response to a compressional point source at depth. Generalized to a pulse, the horizontal and vertical displacements become (with slight notational changes):

$$
\begin{aligned}
& q_{0}=\frac{4 A}{\pi i} \int_{\lambda-i \infty}^{\lambda+i \infty} \frac{e^{s t}}{s+\theta^{-1}} d s \int_{0}^{\infty} \frac{\left(-\alpha_{2}^{\prime} k^{2} \frac{s^{2}}{\beta_{2}^{2}}\right)}{f_{A}(k)} e^{-\alpha_{2} h} J_{1}(k r) d k \\
& w_{0}=\frac{2 A}{\pi i} \int_{\lambda-i \infty}^{\lambda+i \infty} \frac{e^{s t}}{s+\theta^{-1}} d s \int_{0}^{\infty} \frac{k \frac{s^{2}}{\beta_{2}^{2}}\left(2 k^{2}+\frac{s^{2}}{\beta_{2}^{2}}\right)}{f_{A}(k)} e^{-\alpha_{2} h} J_{0}(k r) d k
\end{aligned}
$$

where $f_{A}(s, k)$ is the Rayleigh function in (4). These expressions have the form of (1). (14) results immediately, with:

$\begin{array}{rrr} & q_{0} & w_{0} \\ N & 1 & 0 \\ N & 2 & 2\end{array}$

There are two relevant Riemann sheets. When $t>r / \beta_{2}$, we consider the sheet $(++)$. When $r / \beta_{2}>t>r / c_{2}$, we consider the sheet $(-+)$. For all nontrivial values of $\rho_{2}, \beta_{2}, c_{2}$, a real root of $f_{A}(c)$ exists on the $(++)$ sheet with a velocity around $0.92 \beta_{2}$, the familiar Rayleigh wave. A pair of roots exists on the $(-+)$ 
sheet. When $\beta_{2} / c_{2}$ is less than 0.52 , these roots are real, unequal, and their velocities are greater than $c_{2}$; hence they do not contribute to the observed signal. For small values of $\beta_{2}$, the roots become complex conjugates, with velocities less than $c_{2}$ and greater than $\beta_{2}$. Since only roots with positive imaginary parts (on the lower sheets) are pertinent to our solution, a single pulse is predicted, arriving between the $P$ and $S$ waves. Since this pulse is trapped with respect to compressional motion and appears shortly after the $P$ wave, we call it the Pseudo- $P$ wave. Figure 6 is the arrival-time diagram showing Pseudo- $P$ and the Rayleigh pulse, normalized to the arrival of the shear wave.

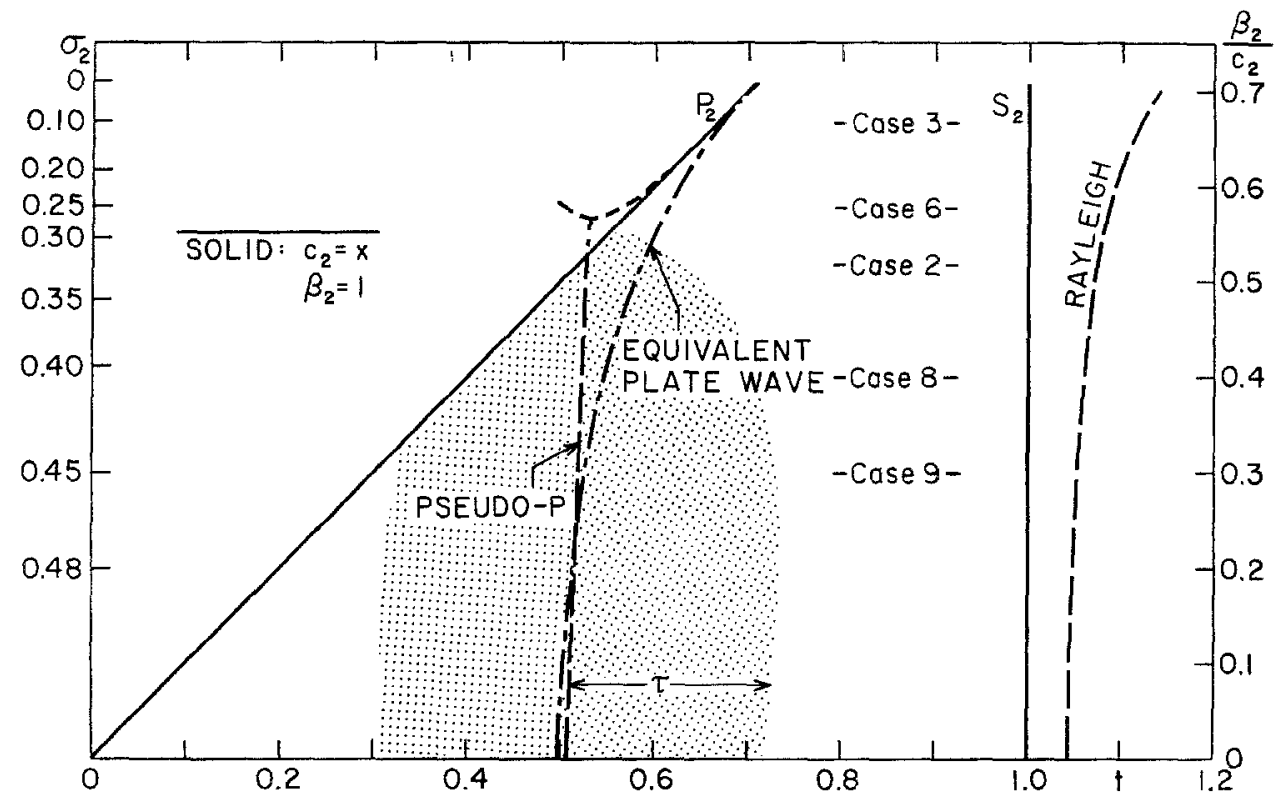

Fig. 6. Normalized arrival time diagram for Lamb's problem. Effect of variable $\beta_{2} / c_{2}$. (Note added in proof: in fig. 6, "Case 3" should read "Case 5.")

The shaded area on the figure represents roughly the extent of the Pseudo- $P$ pulse. When $0.3<\sigma_{2}<0.45$, it appears as an appendage to the $P$-wave. When $\sigma_{2}>0.45$ it becomes distinct from both $P$ and $S$; as $\sigma_{2} \rightarrow 0.50$ (letting $c_{2}$ stay fixed as $\beta_{2} \rightarrow 0$ ), the amplitude of Pseudo- $P$ approaches zero, due to the very great time of arrival relative to the $P$ wave. When $\sigma_{2}<0.30$ no pulse occurs, although the images of the root are plotted using a dashed line. A familiar result states that, when $\sigma_{2}=0.25$, two "extraneous" real roots are found on the lower Riemann sheet. These have been interpreted as describing the angles at which shear waves are converted wholly into $P$-waves upon reflection by the free surface (or vice versa). We may generalize this interpretation: when $\sigma_{2}>0.30$, a shear wave incident on the free surface with a certain "complex angle of incidence" is converted entirely into a compressional motion critically refracted along the interface. Conversely a signal moving along the interface with velocity near $c_{2}$ (making it largely a $P$-wave) continually feeds a shear wave radiating into the solid. If we 
assume a pulse moving along the surface at a velocity between $\beta_{2}$ and $c_{2}$, then only radiating shear waves are possible. The particular velocity of pseudo- $P$ is that velocity which minimizes the loss by conversion to shear waves.

Gilbert and Laster have found Pseudo- $P$ by evaluating the exact (Cagniard) closed form solution of Lamb's problem. In that framework the signal at times between the arrival of $P$ and $S$ is obtained by evaluating a certain function at a point near the branch cut. When $\sigma_{2}<0.30$, a pole lying on the lower sheet becomes so disposed with respect to the branch cut that it affects the value of the solution for certain values of time. The authors have reported a broad, ill-defined pulse coming after the $P$-wave, a result in agreement with the results reported here (Fig. 6). The method described in the present paper is valid only at long ranges and lacks the precision of the Cagniard method, but it gives us a simple tool for investigating the physical importance of the complex poles.

We do not claim that Pseudo- $P$ would be an observed phase in field problems. It should be observed in model experiments, if sufficient control of the properties is possible. The physical importance of this wave is due to the effect it has on the leaking mode propagation in more elaborate waveguides. In a recent paper (Phinney, 1961), the leaking modes of a liquid layer coupled to a solid halfspace were determined. It was then noticed that the frequency of the first $P L$ mode is somewhat less than expected on the basis of a simple ray theory. This effect becomes more pronounced as the shear velocity of the solid decreases. At the time it was hypothesized that this was due to coupling of the channel wave to an intrinsic long-period vibration of the halfspace. In this paper we have already shown that such a vibration does exist and that it propagates at velocities in common with the $P L$ group velocity $\left(\beta_{2}<e<c_{2}\right)$.

The coupling effect is demonstrated by computing $P L$ wave dispersion for several cases whose properties resemble those which permit propagation of Pseudo- $P$. The following models are presented in Fig. 7 .

$\begin{array}{clll}\text { CASE } & \sigma_{2} & \beta_{2} / c_{2} & \\ 5 & 0.10 & 0.667 & \\ 6 & 0.25 & 0.577 & \rho_{2}=2 \rho_{1} \\ 2 & 0.32 & 0.517 & \\ 8 & 0.405 & 0.400 & c_{2}=3 c_{1} \\ 9 & 0.45 & 0.300 & \end{array}$

The equivalent Lamb problems are indicated on Fig. 6 by case number. On Fig. 7 the arrows labeled (2), (8), and (9) represent the velocity of pseudo-P in the halfspace according to Fig. 6 . For cases 5 and 6 the pseudo- $P$ root is degenerate and is not plotted; although the peak does not propagate in such cases, the tail of the pulse falls in the range $\left(\beta_{2}<r / t<c_{2}\right)$ and the corresponding $P L$ mode is affected in the immediate neighborhood of the $P$-arrival. Since all systems of geophysical interest are layered (or worse), we conclude that Pseudo- $P$ manifests itself indirectly, by coupling to leaking modal oscillations of a layered system.

We may test the physical meaning of waves such as Pseudo- $P$ in another way. This wave is presumably coupled to shear waves radiating into the halfspace; it therefore loses energy by leakage. If this is correct, then we may stop the leakage 
by putting a perfectly reflecting bottom on the structure to trap the downward radiating shear waves. The perfectly trapped (normal) modes of a free elastic plate should then show coupling to the pseudo- $P$ wave of an elastic halfspace. At

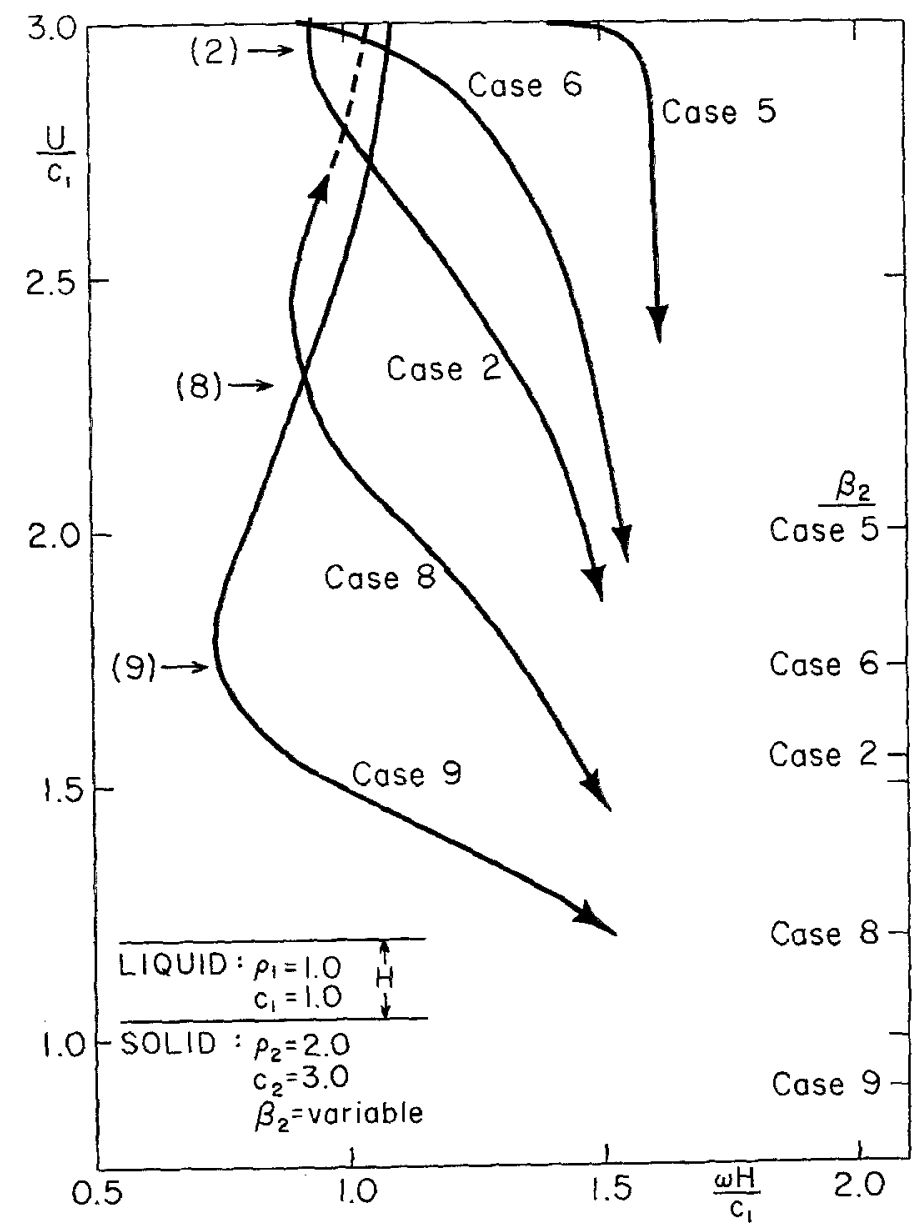

Fig. 7, Coupling of Pseudo- $P$ to group velocity of first PL mode: liquid layer overlying a solid half space. Effect of variable $\beta_{2} / c_{2}$. Pseudo-P velocities for Cases $2,8,9$ shown by arrows.

long wave-lengths the appropriate wave is the extensional, or plate wave, whose velocity of propagation is given by the formula:

$$
\frac{c_{P}}{\beta_{2}}=2 \sqrt{1-\frac{\beta_{2}^{2}}{c_{2}^{2}}}
$$

This has been evaluated for various Poisson constants and plotted as a normalized arrival time in Fig. 6 . The agreement of the plate wave with the Pseudo- $P$ wave is very good in the range where the latter exists.

Recent work on early-arriving waves, which are usually leaking waves, has tended to associate them with progressive elliptical surface motion as contrasted with the 
retrograde motion of Rayleigh waves. Oceanic and continental $P L$ waves, as well as the plate wave just cited, fall into this class. Typical numerical results pertinent to the Pseudo- $P$ have been applied to (14) with the object of estimating the sense of the orbital ellipse. A rather flat prograde ellipse is found, having its major axis tipped backward about 45 degrees to the vertical. We may thus think of Pseudo- $P$ as the basic progressive elliptical motion of a halfspace, dual to the Rayleigh motion. It manifests itself chiefly in similar motions of more complicated layered waveguides.

\section{The Liquid/Solid Interfact Problem}

Having introduced the pseudo- $P$ pulse, we are in a position to consider more complicated problems; we expect to find generalizations of the pseudo- $P$ and Rayleigh waves, as well as to encounter the familiar Stoneley wave. In an earlier section, numerical data for this problem were used in a discussion of the behavior of (14). A complete physical discussion as well as representative numerical results are now offered.

The Stoneley wave, which has a velocity less than the least body wave velocity (usually the liquid velocity), propagates as a trapped wave with respect to all modes of motion. As is well known, the Stoneley wave exists for all non-trivial values of the wave velocities, and has been verified experimentally in recent model work (Roever and Vining, 1959; and Osborne and Hart, 1945). If we perturb Lamb's problem by imposing a liquid halfspace on the surface, the Rayleigh wave velocity will, for a great many cases, be greater than the compressional wave velocity in the liquid. The Rayleigh wave thus excites a radiating $P$-wave in the liquid, which serves to abstract energy from the interface. This leaking Rayleigh wave, or pseudo-Rayleigh wave, has been thoroughly discussed by Strick (1959) in the context of the closed algebraic solution (Cagniard). In our earlier discussion of the pulse solution (14) we have verified most of the properties discussed by Strick. The pseudo-Rayleigh wave is the least damped, and hence the most observable, of all the leaking wave types which arise from lower sheet roots of the characteristic function. In the numerical results schematically shown in Figs. 8-13, the pseudoRayleigh wave is shown arriving directly after the $S$-wave with a pulse width which involves the initial $S$-motion. When the shear velocity is nearly the same as the liquid velocity, the pseudo-Rayleigh wave ceases to exist. Whenever $c_{1}>\beta_{2}$ the Rayleigh motion is manifest in the Stoneley wave.

It is to be noted that seeming disagreement exists with a result in Strick's paper. On our figures, the pseudo-Rayleigh velocity is equal to the true Rayleigh velocity (Fig. 6) asymptotically near $\sigma_{2}=0$, with considerable disagreement as $\beta_{2} \rightarrow c_{1}$. Strick showed that the two velocities were always the same. We have defined the pulse velocity by the velocity of the pulse envelope maximum, while Strick defines it as the velocity of a zero-crossing in the pulse. Thus no contradiction actually exists. Detailed questions about the signal if $\beta_{2}$ is close to $c_{1}$ must be resolved by numerical evaluation of the closed form solution.

The generalization, for the liquid/solid interface, of the pseudo- $P$ pulse is shown in Fig. 8-13. The pole is located on the Riemann sheet appropriate to radiation as shear waves and as $P$-waves in the liquid, but describing trapped $P$-waves in the 


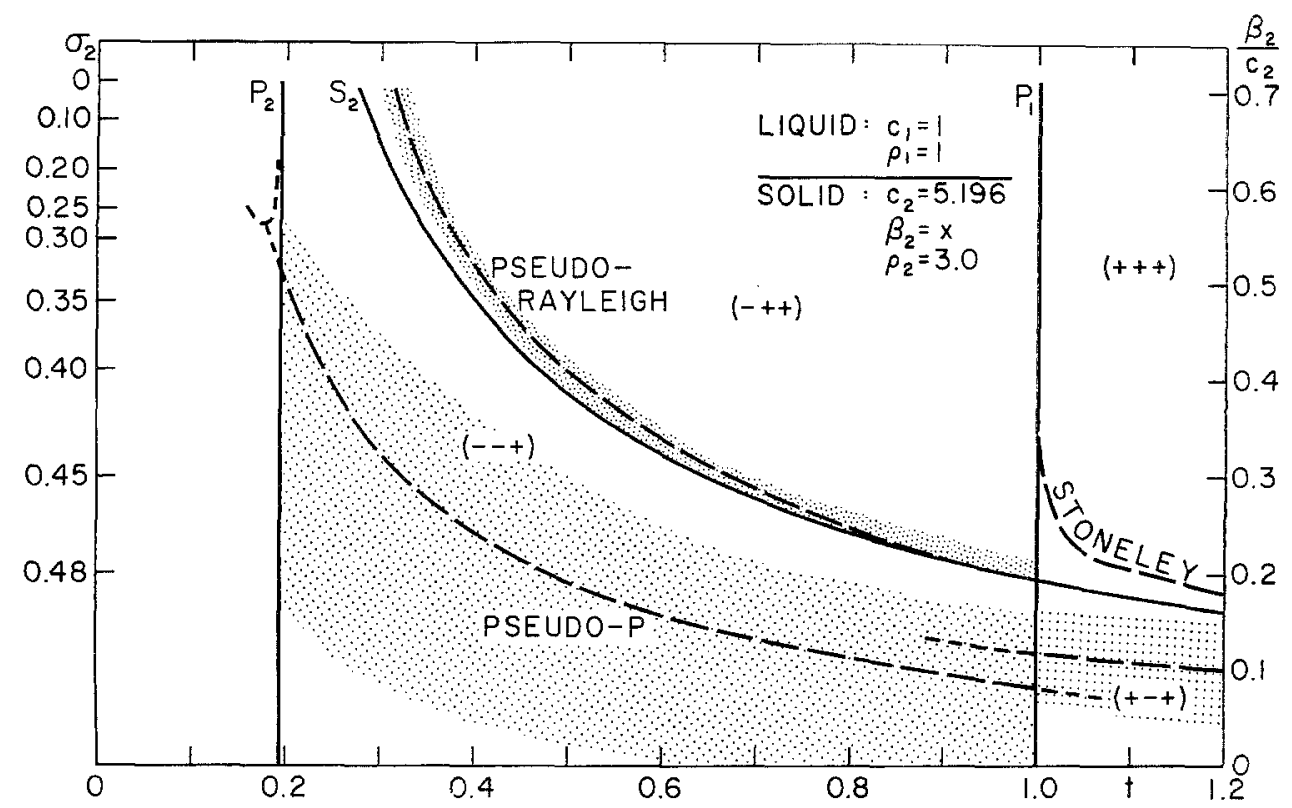

FIG. 8. Normalized arrival time diagram for liquid/solid interface. Effect of variable $\beta_{2} / c_{2}$. $c_{2} / c_{1}=\tilde{5} .196$.

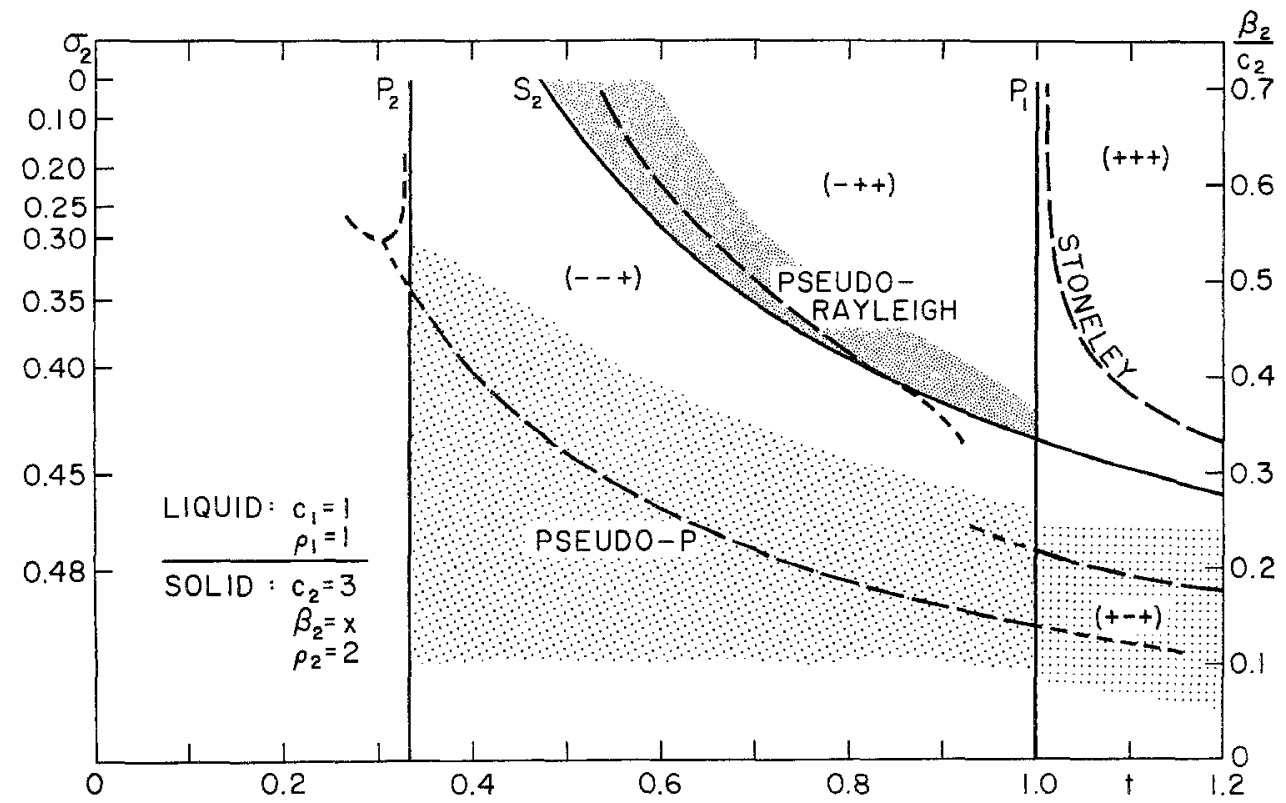

FIG. 9. Normalized arrival time diagram for liquid/solid interface. Effect of variable $\beta_{2} / c_{2}$. $c_{2} / c_{1}=3$.

solid, namely the sheet $(--+)$. The figures show that pseudo- $P$ is little affected by the liquid when a poor impedance match occurs between the media. When one or both of the solid velocities is near to or less than $c_{1}$, pseudo- $P$ differs considerably in breadth and velocity from the "standard" in Lamb's problem. 


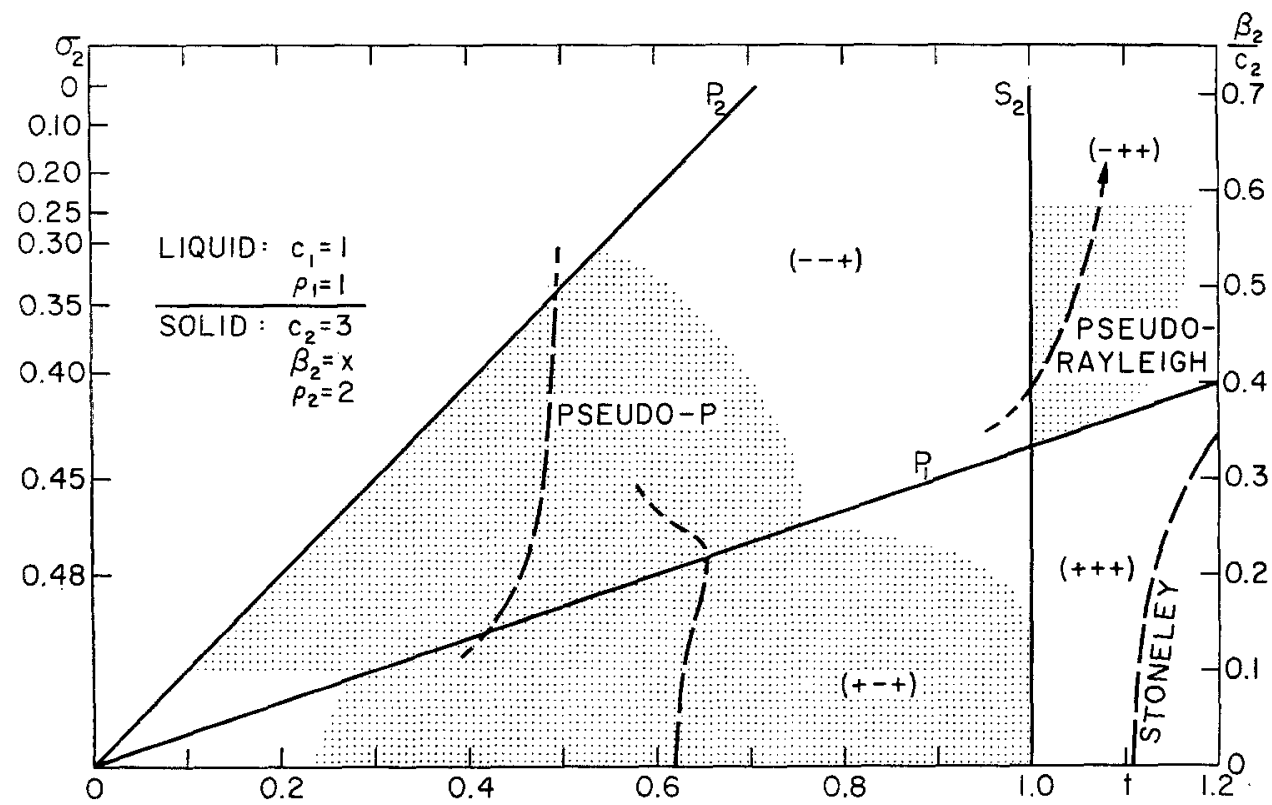

FIg. 10. Arrival time diagram of Fig. 9 renormalized to arrival of the shear wave.

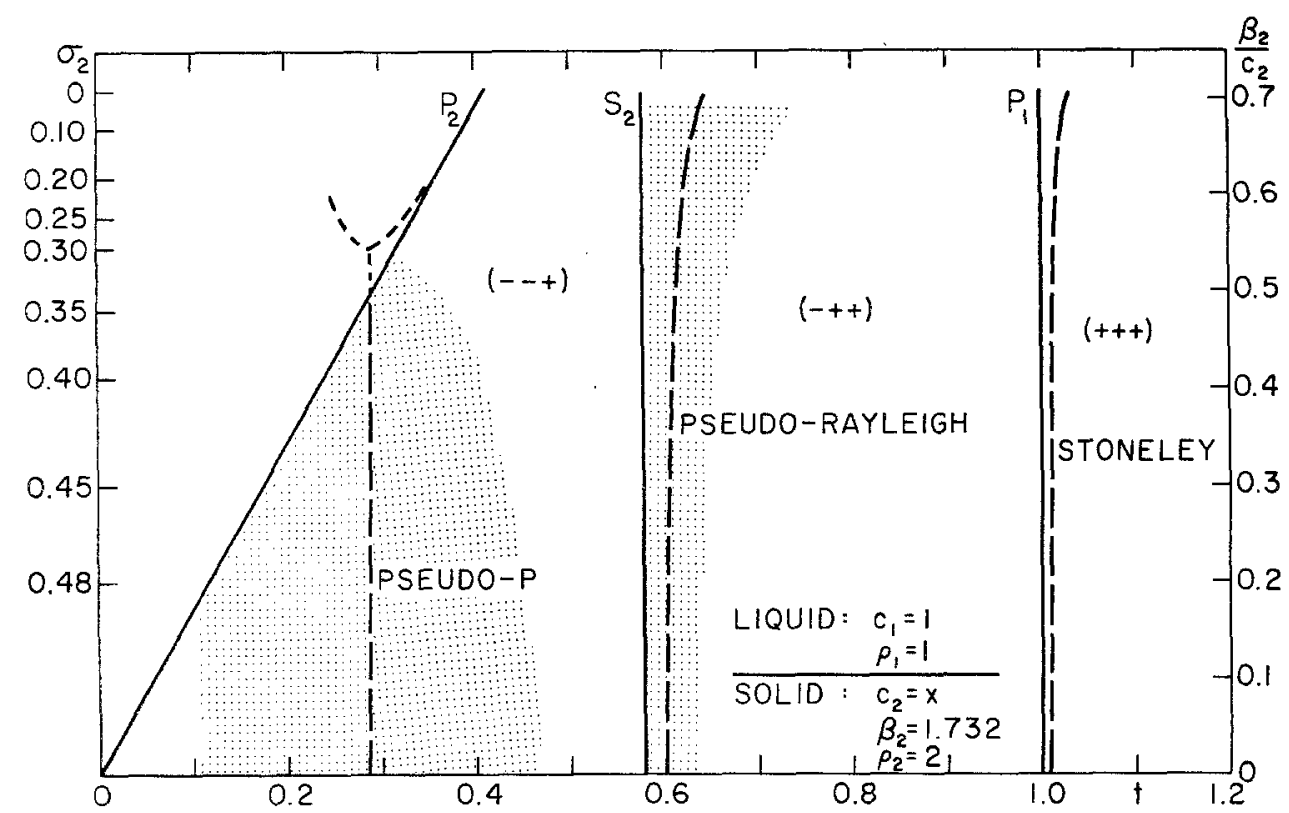

FIg. 11. Normalized arrival time diagram for variable $c_{2}$

If $\beta_{2}<c_{1}$, the sheet $(+-+)$ is relevant to the motion when $r / \beta_{2}>t>r / c_{1}$. It is seen in the figures that a root occurs with the velocity roughly $1.4-1.5$ times the shear velocity. The pulse breadth (17) is so great that we can hardly regard this wave as easily observable. Its amplitude must be quite small in view of the long times at which it arrives. We suggest that it may be a manifestation of pseudo- $P$ 


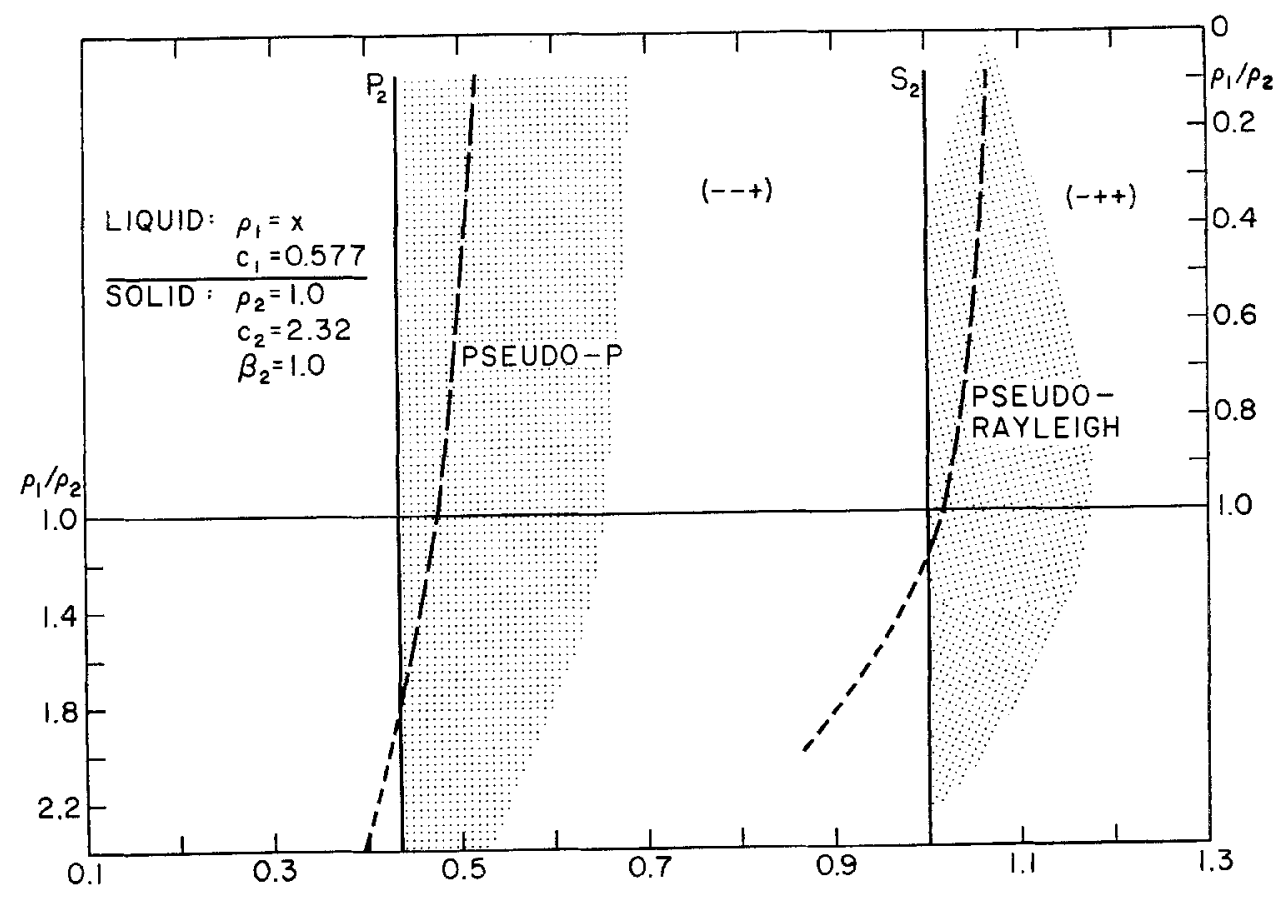

FIG. 12. Normalized arrival time diagram for variable density contrast.

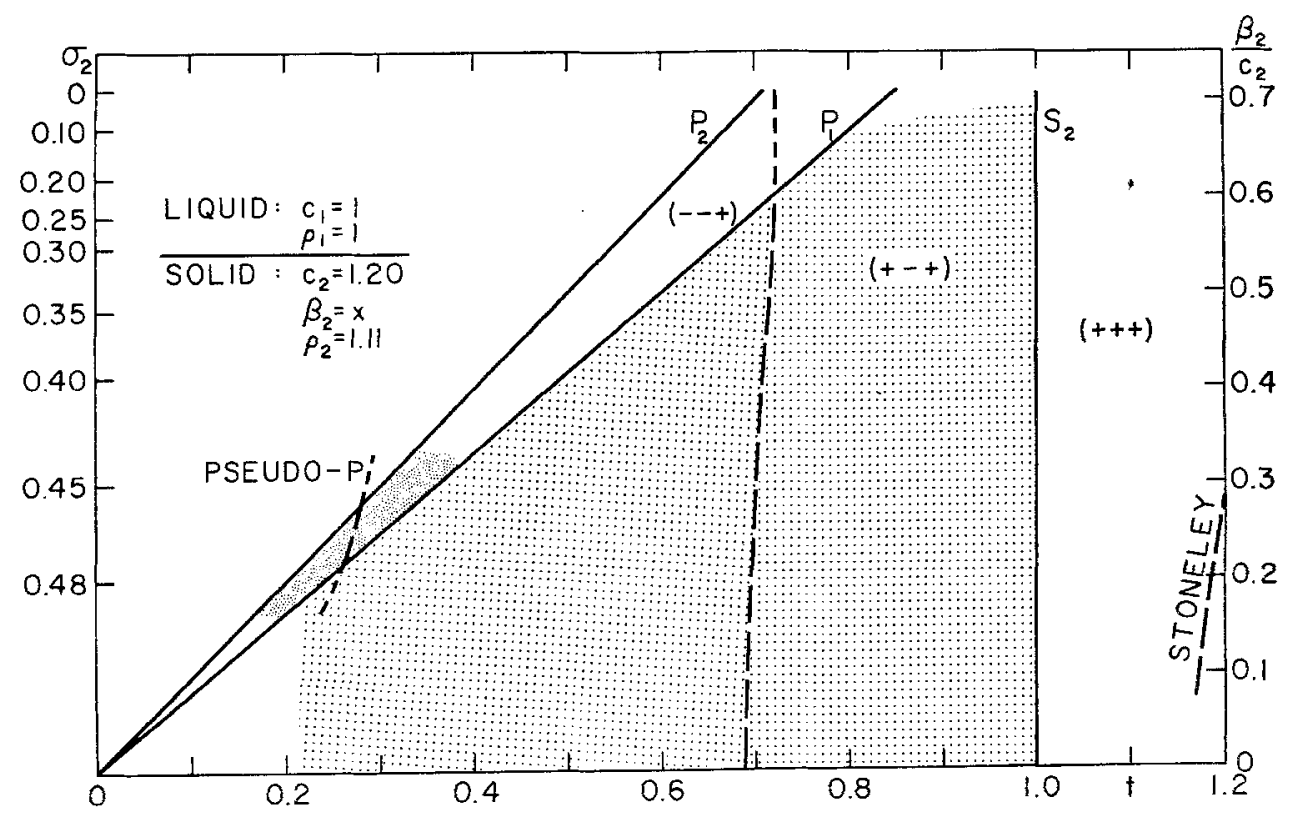

Frg. 13. Normalized arrival time diagram for liquid/solid interface. Effect of variable $\beta_{2} / c_{2} \cdot c_{2} / c_{1}=1.20$. 
motion, much as the Rayleigh motion is described by two different branches, depending as $c_{1}<\beta_{2}$ or vice versa. This is probably the same root encountered by Strick, by a zero-crossing formula, with a velocity $\sqrt{2}$ times the shear velocity.

Figure 12 deserves special comment. It shows the effect on the pseudo- $P$ and pseudo-Rayleigh waves of varying the density ratio. The ratio $\beta_{2} / c_{2}=0.43$ is chosen to insure that pseudo- $P$ is not degenerate. When $\rho_{1}$ becomes greater than $\rho_{2}$, the increased impedance of the liquid rapidly suppresses the free motion of the solid. The effect is less drastic on pseudo- $P$ than on pseudo-Rayleigh, since the former is largely a compressional motion parallel to the interface and involves less coupling to the liquid.

\section{The Solid/Solid Interface Problem}

Numerical results for this problem will be reported in a separate paper. Due to the increased number of parameters involved, many figures will be required to even outline the general behavior of the pseudo-waves. The following general results may be mentioned here.

If the slow halfspace is viewed as a perturbation of the faster medium, we find pseudo- $P$ and pseudo-Rayleigh coupled to the $P$ and $S$ waves of the faster medium, with radiation into the slower material. If the faster medium is viewed as a perturbation of the slower, we find that it suppresses the pseudo-waves in the slower material, although roots may be found which are trivial in the sense that they do not contribute appreciably to the signal. The true Stoneley wave for this problem appears as a very special case of the pseudo-Rayleigh wave in the faster medium, in the event that its velocity turns out to be slower than all four body wave velocities. Gilbert and Laster (1961) have treated this problem from the point of view of the exact (Cagniard) solution, and our numerical results should provide a valuable complement to their detailed discussion.

\section{APPENDIX}

It is easily shown that the exact closed form solution by Cagniard's method yields restrictions on pole contributions from the lower Riemann sheets analogous to those found by the more traditional analysis (Table 2). To simplify the analysis, we set $h=z=0$. This method is, of course, applicable only to the unlayered, simple interface problem.

The Fourier transform of $x(t)$ may be reduced to a one-sided transform by virtue of $x(t)$ vanishing prior to $t=0$ :

$$
\bar{x}=\int_{-\infty}^{\infty} x(t) e^{-i \omega t} d t=\int_{0}^{\infty} x(t) e^{-i \omega t} d t
$$

Setting up steady state solutions of the wave equation which satisfy the boundary conditions on the interface, we obtain:

$$
\bar{x}=A S(\omega) \int_{0}^{\infty} \frac{g(\omega, k)}{f(\omega, k)} k J_{p}(k r) d k
$$

where $S(\omega)$ is the Fourier transform of the source time dependence. 
We now consider the solution at sufficiently long range that we may approximate the Bessel function and drop the inward traveling wave.

$$
J_{p}(k r)=H_{p}^{(1)}(k r)+H_{p}^{(2)}(k r) \rightarrow H_{p}^{(2)}(k r) \sim \sqrt{\frac{2}{\pi k r}} \exp [-i(k r-\pi / 4-p \pi / 2)]
$$

Then:

$$
\bar{x}=A S(\omega) e^{1 / 2(\pi i)[p+(1 / 2)]} \sqrt{\frac{2}{\pi r}} \int_{0}^{\infty} \frac{g(\omega, k)}{f(\omega, k)} k^{1 / 2} e^{-i k r} d k
$$

If we set $u=k / \omega$, then $d k=\omega d u$ and:

$$
\bar{x}=A S(\omega) e^{1 / 2(\pi i)[p+(1 / 2)]} \sqrt{2 / \pi r} \int_{0}^{\infty} \frac{g(\omega, u \omega)}{f(\omega, u \omega)} \sqrt{u \omega} e^{-i u \omega r} \omega d u
$$

Now set $\tau=u r: d u=d \tau / r$ :

$$
\bar{x}=A S(\omega) e^{1 / 2(\pi i)[p+(1 / 2)]} \sqrt{2 / \pi r} \int_{0}^{\infty} \frac{g(\omega, \omega \tau / r)}{\overline{f(\omega, \omega \tau / r)}}\left(\frac{\omega}{r}\right)^{3 / 2} \tau^{1 / 2} e^{-i \pi \tau} d \tau
$$

For solvable cases (simple interface problem), $g$ and $f$ are homogeneous in $\omega$. If $f$ and $g$ are now factored, the resulting power of $\omega$ will just be the difference in homogeneous degree of $f$ and $g$. We set this equal to $N-2$ to conform with the convention used in the main text.

$$
\bar{x}=A S(\omega) e^{1 / 2(\pi i)[p+(1 / 2)]} \sqrt{2 / \pi} \omega^{N-2} \omega^{3 / 2} r^{-2} \int_{0}^{\infty} \frac{g(1, \tau / r)}{f(1, \tau / r)} \tau^{1 / 2} e^{-i \omega \tau} d \tau
$$

When $S(\omega)=1$, the source is a delta function; when $S(\omega)=1 / i \omega$, the source is a unit step. By taking $S(\omega)=1 / \sqrt{i \omega}$, we simplify the analysis as well as represent a source somewhere between the step and delta function.

$$
\bar{x}=A \sqrt{2 / \pi} e^{1 / 2(p \pi i)} \omega^{N-1} r^{-2} \int_{0}^{\infty} \frac{g(1, \tau / r)}{f(1, \tau / r)} \tau^{1 / 2} e^{-i \omega \tau} d \tau
$$

The time function corresponding to $\bar{x}$ is now obtained by inspection:

$$
x=A \sqrt{2 / \pi} e^{1 / 2(p \pi i)} r^{-2} e^{(-1 / 2)(N-1) \pi i} \frac{d^{N-1}}{d t^{\bar{N}-1}}\left\{t^{1 / 2} \frac{g(1, t / r)}{f(1, t / r)}\right\}
$$

where the variable $t$ runs from 0 to $\infty$ along the real $\tau$ axis.

The radicals $\nu_{i}$ generating branch cuts must be expressed in terms of $\tau$.

$$
\nu_{i}=\sqrt{k^{2}-\frac{\omega^{2}}{v_{i}^{2}}}=\sqrt{u^{2} \omega^{2}-\frac{\omega^{2}}{v_{i}^{2}}}=\omega \sqrt{\frac{t^{2}}{r^{2}}-\frac{1}{v_{i}^{2}}}=\omega \nu_{i}^{\dagger}
$$




$$
\tau \text {-plane }
$$

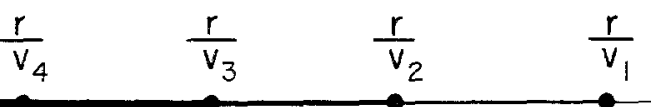

FIG. 14. r-plane, showing branch euts according to convention.

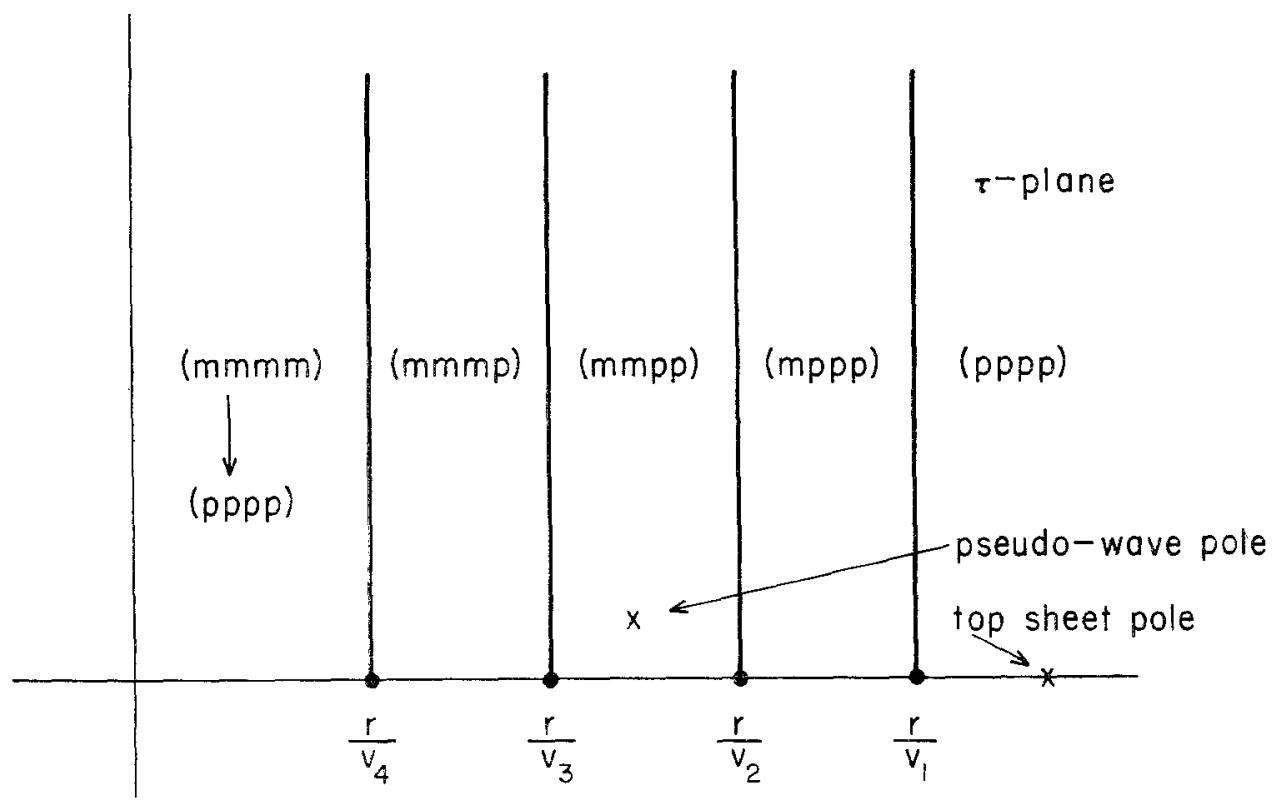

Fig. 15. $\tau$-plane, with branch cuts redefined to show effect of lower sheet poles on the response when $\tau=t=$ real.

If the Riemann sheets are defined as before, with $\operatorname{Re} \nu_{i}=0$ defining the cuts, we may locate the singularities in the $\tau$-plane (Fig. 14). Here $t$ is assumed to lie along the bottom bank of the eut.

When $t$ lies between two of the branch points in the $\tau$-plane, the value of $g / f$ is affected by any poles near $\tau=t$. There are none on the top sheet except the surface 
wave pole lying on the real axis at $\tau>r / v_{1}$. There may be poles on lower sheets, however, and one which is contiguous to the point $t$ will affect the signal in that range. This is made clear by redrawing the branch cuts so as to expose those portions of the lower sheets which represent a continuation of the real $\tau$-axis into the first quadrant. Portions of the $\tau$-plane (in Figure 15) so cut which do not lie on the first sheet are indicated by the conventional notation $(m p p p),(m m p p), \ldots$ etc. Note that for the interface wave problem ( $m m m m)$ and ( $p p p p)$ have the same poles.

The result demonstrated in the previous appendix and used in the main text is now apparent from inspection of the figure. When $t$ lies between the arrival times of two body waves, the signal may be influenced by zeros of $f$ lying on the Riemann sheet contiguous to $t$. This is the same conclusion obtained by the longer analysis based on the straightforward evaluation of the double integral form of the solution. Table 2 in the main text will be seen to be equivalent to the results demonstrated graphically in Fig. 15.

We might write down a formula which isolates the effect of such a lower sheet pole in the $\tau$-plane and attempt a close comparison with the pulse expression (14) obtained in the main text. The difference between the two representations is too great, however: In the $\tau$-plane, proximity of the branch point manifests itself by multiplying the effect of the pole. The analysis in this paper leading up to (14) separates the pole contributions from the body waves in such a way that they add to give the total signal.

\section{ACKNOWLEDGMENTS}

The author wishes to thank Dr. Frank Press for his advice and encouragement throughout this work.

This research was partially supported by Contract No. DA-04-495 Ord. 1808 (Model Seismology) of the Office of Ordnance Research, U. S. Army.

The author did this work under a National Science Foundation Fellowship for 1960-1961.

\section{REFERENCES}

Cagniard, L.

1939. Réflexion et Réfraction des ondes Séismique Progressives, Gauthier-Villars, Paris. $255 \mathrm{pp}$.

Ewing, W. M., W. S. Jardetzky, and F. Press

1957. Elastic Waves in Layered Media, MeGraw-Hill, New York. 379 pp.

Garvin, W. W.

1956. "Exact Transient Solution of the Buried Line Source Problem," Proc. Roy. Soc. A., 234: 528-541.

Gilbert, F.

1956. Seismic Wave Propagation in a Two-Layer Half-Space, Ph.D. thesis, Massachusetts Institute of Technology.

Gilbert, F., and S. L. Laster

1961. "Excitation and Propagation of Pulses on an Interface," Bull. Seism. Soc. Am., (in press).

Ginzbarg, A. S., and E. Strick

1958. "Stoneley-Wave Velocities for a Solid-Solid Interface," Bull. Seism. Soc. Am., 48 : 51.

Osborne, M. F. M., and S. O. Hart

1945. "Transmission, Reflection, and Guiding of an Exponential Pulse by a Steel Plate in Water, I Theory," Jour. Acoust. Soc. Am., 17: 1-18. 
Phinney, R. A.

1961. "Leaking Modes in the Crustal Waveguide, Part I: The Oceanic PL Wave," Jour. Geophys. Res., 66: 1445-1470.

Roever, W. L., and T. F. Vining

1959. "Propagation of Elastic Wave Motion from an Impulsive Source Along a Fluid-Solid Interface. I Experimental Pressure Response," Phil. Trans. Roy. Soc. A., 251: 455-465.

Rosenbaum, J. H.

1960. "The Long-Time Response of a Layered Elastic Medium to Explosive Sound," Jour. Geophys. Res., 65: 1577-1614.

Scholte, J. G.

1949. "On True and Pseudo-Rayleigh Waves," Proc. Kon. Ned. Akad. Wetensch. Amst., $52: 652-653$.

Stoneley, R.

1924. "Elastic Waves at the Surface of Separation of Two Solids," Proc. Roy. Soc. A., 106: $416-428$.

Strick, E.

1959. "Propagation of Elastic Wave Motion from an Impulsive Source Along a Fluid-Solid Interface. Parts II and III," Phil. Trans. Roy. Soc. Lon., A, 251: 465-523.

Strick, E., and A. S. Ginzbarg

1956. "Stoneley-Wave Velocities for a Fluid-Solid Interface," Bull. Seism. Soc. Am., 46: 281.

Seismological Laboratory,

California Institute of Technology,

Pasadena, Caltfornta.

(Division of the Geological Sciences, contribution no. 1023.) 